\title{
Adaptations in energy metabolism and gene family expansions revealed by comparative transcriptomics of three Chagas disease triatomine vectors
}

\author{
Jesús Martínez-Barnetche ${ }^{1 \dagger}$, Andrés Lavore ${ }^{2+}$, Melina Beliera², Juan Téllez-Sosa ${ }^{1}$, Federico A. Zumaya-Estrada ${ }^{1}$,
} Victorio Palacio², Ernestina Godoy-Lozano', Rolando Rivera-Pomar ${ }^{2,3}$ and Mario Henry Rodríguez ${ }^{1 *}$

\begin{abstract}
Background: Chagas disease is a parasitic infection caused by Trypanosoma cruzi. It is an important public health problem affecting around seven to eight million people in the Americas. A large number of hematophagous triatomine insect species, occupying diverse natural and human-modified ecological niches transmit this disease. Triatomines are long-living hemipterans that have evolved to explode different habitats to associate with their vertebrate hosts. Understanding the molecular basis of the extreme physiological conditions including starvation tolerance and longevity could provide insights for developing novel control strategies. We describe the normalized cDNA, full body transcriptome analysis of three main vectors in North, Central and South America, Triatoma pallidipennis, T. dimidiata and T. infestans.

Results: Two-thirds of the de novo assembled transcriptomes map to the Rhodnius prolixus genome and proteome. A Triatoma expansion of the calycin family and two types of protease inhibitors, pacifastins and cystatins were identified. A high number of transcriptionally active class I transposable elements was documented in $T$. infestans, compared with T. dimidiata and T. pallidipennis. Sequence identity in Triatoma-R. prolixus 1:1 orthologs revealed high sequence divergence in four enzymes participating in gluconeogenesis, glycogen synthesis and the pentose phosphate pathway, indicating high evolutionary rates of these genes. Also, molecular evidence suggesting positive selection was found for several genes of the oxidative phosphorylation I, III and V complexes.

Conclusions: Protease inhibitors and calycin-coding gene expansions provide insights into rapidly evolving processes of protease regulation and haematophagy. Higher evolutionary rates in enzymes that exert metabolic flux control towards anabolism and evidence for positive selection in oxidative phosphorylation complexes might represent genetic adaptations, possibly related to prolonged starvation, oxidative stress tolerance, longevity, and hematophagy and flight reduction. Overall, this work generated novel hypothesis related to biological adaptations to extreme physiological conditions and diverse ecological niches that sustain Chagas disease transmission.
\end{abstract}

Keywords: Chagas disease, Reduviid bugs, Transcriptome, metabolism, oxidative phosphorylation

\footnotetext{
* Correspondence: mhenry@insp.mx

${ }^{\dagger}$ Equal contributors

${ }^{1}$ Centro de Investigación Sobre Enfermedades Infecciosas, Instituto Nacional

de Salud Pública, Cuernavaca, México

Full list of author information is available at the end of the article
}

(c) The Author(s). 2018 Open Access This article is distributed under the terms of the Creative Commons Attribution 4.0 International License (http://creativecommons.org/licenses/by/4.0/), which permits unrestricted use, distribution, and reproduction in any medium, provided you give appropriate credit to the original author(s) and the source, provide a link to the Creative Commons license, and indicate if changes were made. The Creative Commons Public Domain Dedication waiver (http://creativecommons.org/publicdomain/zero/1.0/) applies to the data made available in this article, unless otherwise stated. 


\section{Background}

Chagas disease is an important neglected tropical disease in Central and South America. The causative agent is the protozoan Trypanosoma cruzi, transmitted to humans by hematophagous insects of the Reduviidae family. Among the Reduviidae, the Triatominae subfamily conforms one of 21 subfamilies. Triatomines are obligatory blood feeders evolved from predatory ancestors. The Rhodniini and Triatomini tribes contain most species within the subfamily. The Rhodniini tribe is an accepted monophyletic group [1], while paraphyly of the Triatomini tribe is in debate $[2,3]$.

With few exceptions, triatomine species occurs in the Americas occupying a large variety of ecological niches. In this complex mosaic of natural and human-modified ecotopes, triatomines have evolved to explode different habitats associated with their vertebrate hosts. Rhodnius species mostly dwell in palm tree canopies were they feed on birds, while Triatoma species are land dwellers, living in crevices and burrows were they feed on birds and mammals [4]. Most triatomines are sylvatic, but they have also adapted to domestic and peridomestic habitats, in close relationship with humans. This extended distribution, their longevity and the ability for vectoring $T$. cruzi result in Chagas disease endemism in Latin America, spanning from Northern México to Southern Argentina with an estimated seven to eight million people infected [5].

Four species are relevant vectors of Chagas disease in the Americas: Rhodnius prolixus, Triatoma dimidiata, $T$. pallidipennis, and $T$. infestans. $R$. prolixus, a model organism for insect physiology, is a main vector in Venezuela, Colombia, Peru and whole Central America [6, 7]. Triatoma dimidiata, consist of several variants occupying sylvatic, peri-domestic and domestic habitats, making a main Chagas disease vector in Central America and Mexico [8-10]. Triatoma pallidipennis stands out by showing the capacity to colonize domestic environments in at least 11 Mexican States with a T. cruzi infection prevalence up to $90 \%[11,12]$. The most widespread Chagas disease vector in South America is $T$. infestans, largely adapted to domiciliary habitats, but with sylvatic populations that repopulate the human environment. This is one of the reasons for failure of the indoor insecticide spraying strategy deployed in South America endemic countries [13].

New strategies for Chagas control are urgently needed; information on the genetic basis of their biological adaptations to extreme physiological conditions such as prolonged starvation periods, their extended life span and their capability to adapt to diverse ecological niches could provide insights for novel control interventions. However, despite of their medical significance, detailed genetic information in triatomines is still scarce. The genome of $R$. prolixus has been sequenced [14], but genomic information of the relevant vectors of the Triatoma genus is still limited to organ-specific transcriptomes or selected gene families [15-21]. Thus, we still lack information about the gene composition in triatomines to compare protein families and biological processes, and, therefore, a comprehensive picture of the vectors physiology and evolution.

Transcriptome analysis of organisms without sequenced genomes provides a preliminary catalogue, useful for gene discovery, including specific molecules and their putative functions for the comparative analysis among related organisms, as well as a means for gene prediction validation in future genome projects. We generated transcriptomes derived from normalized $T$. infestans, $T$. dimidiata and $T$. pallidipennis cDNA libraries from all stages of their life cycle and compared them with the genome of $R$. prolixus. This allowed the identification of a large set of shared genes, gene expansions and higher sequence divergence in energy metabolismrelated genes, possibly related to adaptations to their life styles, providing the basis for a better understanding of triatomine biology and insights for development of novel control strategies.

\section{Results}

\section{Sequencing metrics and assembly}

Totals of 164.6, 112.8 and 202.6 megabases of raw sequence data were generated for $T$. infestans, $T$. dimidiata and $T$. pallidipennis, respectively, which resulted in a cDNA assembly of 3904, 4847 and 5148 isogroups (unigenes) containing from $35 \%$ to $69 \%$ of assembled reads using the Newbler assembler (Table 1). Most isogroups contained a single isotig (transcript isoform). Mean isotig length was 841,840 and 893 bp in comparison to 1017 bp in $R$. prolixus, with a corresponding N50 of 871,880 and $921 \mathrm{bp}$, respectively (Table 1). Given that we used the long read GS FLX+454-Roche system, singletons that mapped with the $R$. prolixus genome or proteome were also included to improve gene discovery. The final dataset included 35,629, 29,024 and 31,175 transcripts for $T$. infestans, $T$. dimidiata and $T$. pallidipennis, respectively. The $\mathrm{G}+\mathrm{C} \%$ in the Triatoma transcriptomes (Table 1) was slightly lower than in $R$. prolixus transcript dataset $(39.84 \pm 6.79 \%)$.

For all datasets, there was significant mitochondrial transcription (4.6-7.5\%), as revealed by transcriptome mapping to the $T$. dimidiata mitochondrial genome [22]. The $T$. dimidiata coverage of the mitochondrial genome was near complete, whereas for $T$. pallidipennis and $T$. infestans $86 \%$ and $46 \%$ of the mitochondrial genome was covered, respectively (Table 2). Nevertheless, mitochondrial coverage in the three species was 
Table 1 Sequencing metrics

\begin{tabular}{|c|c|c|c|c|c|c|}
\hline \multirow{2}{*}{ Raw sequencing } & \multicolumn{2}{|c|}{ T. dimidiata } & \multicolumn{2}{|c|}{ T. infestans } & \multicolumn{2}{|c|}{ T. pallidipennis } \\
\hline & & & & & & \\
\hline Raw reads & 358,962 & & 559,962 & & 626,401 & \\
\hline Filtered reads (PRINTSEQ) & 237,226 & $66.1 \%$ & 347,620 & $62.1 \%$ & 421,667 & $67.3 \%$ \\
\hline Number of bases (Mbps) & 112.8 & & 164.6 & & 202.6 & \\
\hline \multicolumn{7}{|l|}{ Assembly } \\
\hline Aligned reads & 173,525 & $73.3 \%$ & 161,674 & $46.6 \%$ & 353,083 & $83.8 \%$ \\
\hline Aligned bases (Mbps) & 82.3 & $73.1 \%$ & 74.2 & $45.1 \%$ & 167.2 & $82.6 \%$ \\
\hline Assembled reads & 140,289 & $59.3 \%$ & 124,306 & $35.8 \%$ & 291,051 & $69.1 \%$ \\
\hline Partially assembled reads & 33,138 & $14.0 \%$ & 37,334 & $10.8 \%$ & 61,847 & $14.7 \%$ \\
\hline Singletons & 56,397 & $23.8 \%$ & 174,467 & $50.3 \%$ & 54,964 & $13.1 \%$ \\
\hline Isogroups & 4847 & & 3904 & & 5148 & \\
\hline Average contig count & 1.6 & & 1.7 & & 1.8 & \\
\hline Average isotig count & 1.3 & & 1.2 & & 1.3 & \\
\hline Largest isotig count & 15 & & 30 & & 18 & \\
\hline Number of isotigs & 6212 & & 4856 & & 6476 & \\
\hline Average contig count & 1.6 & & 1.5 & & 1.5 & \\
\hline Largest contig count & 7 & & 9 & & 9 & \\
\hline Isotigs with one contig & 3824 & & 3232 & & 4169 & \\
\hline Number of bases (Mbps) & 5.2 & & 4.1 & & 5.8 & \\
\hline Average isotig size & 840 & & 841 & & 893 & \\
\hline N50 isotig size & 880 & & 871 & & 921 & \\
\hline Largest isotig size & 6814 & & 3682 & & 4465 & \\
\hline Q40 plus bases (Mbps) & 3.5 & $95.8 \%$ & 2.9 & $95.9 \%$ & 4.0 & $95.4 \%$ \\
\hline < Q39 bases (Mbps) & 0.2 & $4.2 \%$ & 0.1 & $4.1 \%$ & 0.2 & $4.6 \%$ \\
\hline \multicolumn{7}{|l|}{ full_dataset } \\
\hline Total sequences & 29,024 & & 35,629 & & 31,175 & \\
\hline Contigs/isotigs & 6217 & $21 \%$ & 4886 & $14 \%$ & 6530 & $21 \%$ \\
\hline Singletons & 22,807 & $79 \%$ & 30,743 & $86 \%$ & 24,645 & $79 \%$ \\
\hline Total bases (Mbps): & 16.9 & & 20.9 & & 18.2 & \\
\hline$\% G+C$ & 33.73 & $\pm 5.48 \%$ & 35 & $\pm 6.12 \%$ & 33.7 & $\pm 5.75 \%$ \\
\hline \multicolumn{7}{|l|}{$n r_{\text {_dataset ( }}$ isotig per isogroup) } \\
\hline Total sequences & 27,652 & & 34,646 & & 29,789 & \\
\hline Contigs/isotigs (isotig_dataset) & 4845 & $18 \%$ & 3903 & $11 \%$ & 5144 & $17 \%$ \\
\hline Singletons & 22,807 & $82 \%$ & 30,743 & $89 \%$ & 24,645 & $83 \%$ \\
\hline Total bases (Mbps): & 15.8 & & 20.1 & & 17.1 & \\
\hline
\end{tabular}

sufficient to allow the identification of all the mitochondria-encoded genes involved in oxidative phosphorylation. Only in T. infestans, there was significant transcription (13 K reads, 2.4\%) that covered $100 \%$ of the reported Triatoma virus genome sequence (Table 2).

\section{Comparison to other proteomes}

The number of Triatoma transcripts that bestmatched to the $R$. prolixus predicted peptide dataset were similar among the three species $(\sim 7272$ to
8002 ), which corresponds to $48-53 \%$ of the $R$. prolixus predicted proteome (Table 2). Between 71 and $74 \%$ of the $R$. prolixus proteome had a match (e-value $<1.0 \mathrm{E}-05)$ in the Triatoma transcriptomes. Transcriptome completeness assessment by searching the Core Eukaryotic Genome Dataset (CEGMA) [23] and the Benchmarking Universal Single Copy Orthologs (BUSCO) [24] for the three - Triatoma and R. prolixus revealed very high coverage values. The BUSCO coverage was $82.2 \%$ in $T$. infestans, $85.8 \%$ in $T$. 
Table 2 Transcriptome mapping

\begin{tabular}{|c|c|c|c|c|c|c|}
\hline \multirow{2}{*}{ Match to R. prolixus genome (BLASTN) $)^{b}$} & \multicolumn{2}{|c|}{ T. dimidiata } & \multicolumn{2}{|c|}{ T. infestans } & \multicolumn{2}{|c|}{ T. pallidipennis } \\
\hline & & $\%$ & & $\%$ & & $\%$ \\
\hline Non-redundant Triatoma transcripts matching $R$. prolixus & 17,105 & $61.9 \%$ & 18,074 & $52 \%$ & 17,824 & $60 \%$ \\
\hline Isotigs/contigs ${ }^{a}$ & 3,544 & $73.1 \%$ & 2,459 & $63 \%$ & 3,802 & $74 \%$ \\
\hline Singletons & 13,561 & $59.5 \%$ & 15,615 & $51 \%$ & 14,022 & $57 \%$ \\
\hline \multicolumn{7}{|l|}{ Match to $R$. prolixus proteome $(\mathrm{BLASTX})^{c}$} \\
\hline R. prolixus proteins (best-hit) & 7,865 & $52 \%$ & 7,272 & $48 \%$ & 8,002 & $53 \%$ \\
\hline Pair wise identity & & $78 \%$ & & $79 \%$ & & $79 \%$ \\
\hline R. prolixus proteins (e-value $<1.0 \mathrm{E}-05$ ) & 11,136 & $74 \%$ & 10,674 & $71 \%$ & 10,921 & $72 \%$ \\
\hline Triatoma transcripts matching R. prolixus (e-value $<1.0 \mathrm{E}-05$ ) & 17,576 & $64 \%$ & 18,836 & $54 \%$ & 18,245 & $61 \%$ \\
\hline BUSCO $^{d}$ & 2,297 & $85.8 \%$ & 2,201 & $82.2 \%$ & 2,326 & $86.9 \%$ \\
\hline $\mathrm{CEG}^{\mathrm{e}}$ & 432 & $94.3 \%$ & 415 & $90.6 \%$ & 436 & $95.2 \%$ \\
\hline \multicolumn{7}{|l|}{ T. dimidiata Mitochondrial genome } \\
\hline Mapped reads & 26,670 & $7.4 \%$ & 25,714 & $4.6 \%$ & 47,131 & $7.5 \%$ \\
\hline Coverage (bp) & 16,309 & $95.8 \%$ & 7,937 & $46.6 \%$ & 14,675 & $86.2 \%$ \\
\hline \multicolumn{7}{|l|}{ Triatoma virus } \\
\hline Mapped reads & 31 & $0.01 \%$ & 13,301 & $2.4 \%$ & 8 & $0.0 \%$ \\
\hline Coverage (bp) & 3,234 & $35.8 \%$ & 9,012 & $100.0 \%$ & 1303 & $14.4 \%$ \\
\hline
\end{tabular}

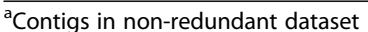

${ }^{b}$ Rhodnius-prolixus-CDC_SCAFFOLDS_RproC3.fa

'Rhodnius-prolixus-CDC_PEPTIDES_RproC3.1.fa . 15,078 proteins

${ }^{d}$ hmmsearch score $\geq 40 ; n=2676$ BUSCO's. $R$. prolixus 2597/2676. 97.0\%

ehmmsearch score $\geq 40 ; n=458$ CEG's. $R$. prolixus $448 / 458$. 97.8

dimidiata and $86.9 \%$ in $T$. pallidipennis, while these coverage values were higher than $90 \%$ for the CEGMA in the three species (Table 2). These metrics indicate that although our datasets do not comprise all the gene content of each species, it is sufficient for a useful approximation for transcriptome to genome gene content comparison.
Each Triatoma transcriptome covers between 20 to $55 \%$ of the proteome of other insects (Table 3). Low proteome coverage was observed for Drosophila melanogaster, with a high-quality annotated proteome, Ixodes scapularis, and Acyrtosiphon pisum. BLAST best-hit protein identity was on average around 55\% and correlated with the phylogenetic distance, such

Table 3 BLASTP comparison of the three Triatoma translated transcriptomes with arthropod proteomes

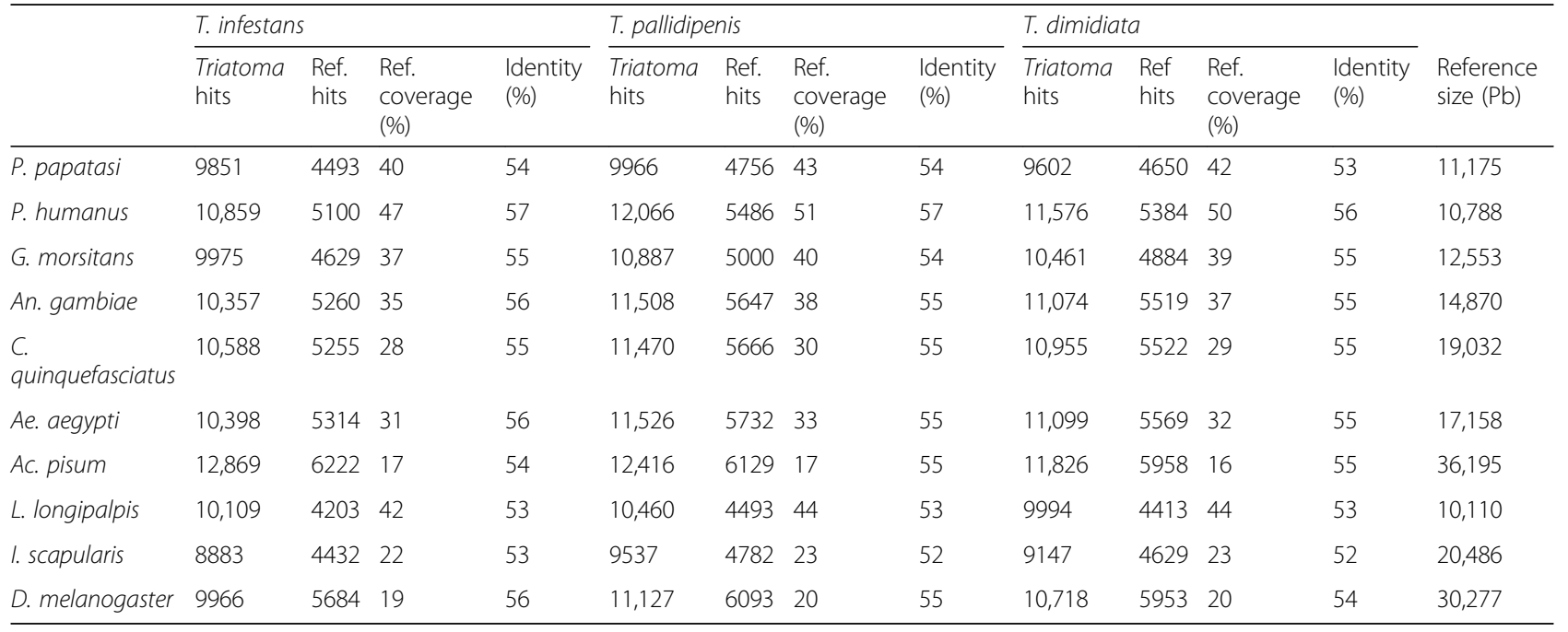


that average protein identity between the three - Triatoma and $R$. prolixus was $\sim 78 \%$ (Table 2 ).

\section{Orthologous genes within $R$. prolixus and Triatoma}

A total of 4054 orthologs were shared (BLAST best reciprocal hits) among the three - Triatoma and $R$. prolixus. A set of 5215 orthologs were present only in the Triatoma sp. studied here. In accordance to the phylogenetic distance, $T$. dimidiata and T. pallidipennis had 10,154 best BLAST reciprocal hits, while the best reciprocal hits among the other triatomines was lower (Fig. 1).

\section{Gene ontology (GO) annotation}

The functional composition of each transcriptome can be influenced by biological variables (tissue type, ontogenic stage, gene content, genetic variation, transcription rate, among others), as well as for technical variables (tissue composition in the library, sequencing depth, normalization process, among others). To assess transcriptional representation bias, we tested Gene Ontology annotated transcriptomes for enrichment compared to the $R$. prolixus predicted proteome. Despite library normalization, the Triatoma transcriptomes were consistently enriched for transcripts involved in core cellular functions, such as protein translation, folding and sorting, generation of precursor metabolites and energy, mitochondrial and ribosomal organization and assembly (Table 4). In contrast, transcripts related to cell signaling, neurological processes, transcription and cell surface proteins were underrepresented in all Triatoma transcriptomes.
We next asked if there was biased transcriptional representation according to GO within the three - Triatoma transcriptomes. In the $T$. infestans transcriptome, we found overrepresentation of the GO term "symbiosis, encompassing mutualism through parasitism" (GO: 0044403). No significant enrichment was found within the $T$. dimidiata and $T$. pallidipennis transcriptomes. The biased transcriptional representation found in the Triatoma datasets, using the $R$. prolixus as reference dataset, may result from transcriptional abundance of core cellular processes and the underrepresentation of certain organs/tissues, such as nervous system and sensory organs during library preparation, and may not reflect accurately gene content. Moreover, the lack of significant enrichment within Triatoma datasets indicates that the source of bias may be predominantly due to transcriptional abundance, and in a lesser extent to differences in gene content, with little participation of technical variation during library preparation.

\section{Protein family overrepresentation}

To assess potential gene expansions, transcriptomes were annotated using InterProScan [25] through the BLAST2GO Pro interface [26]. 17.9, 24.2 and $22.8 \%$ of the respective $T$. infestans, $T$. dimidiata, $T$. pallidipennis non-redundant dataset, and $59.6 \%$ of the $R$. prolixus transcript dataset had at least one InterPro annotation. A Fisher's exact test was performed to identify enrichment of InterPro entries in the test transcriptome isotig dataset (1 isotig per isogroup, excluding singletons) compared with the $R$. prolixus transcript dataset as

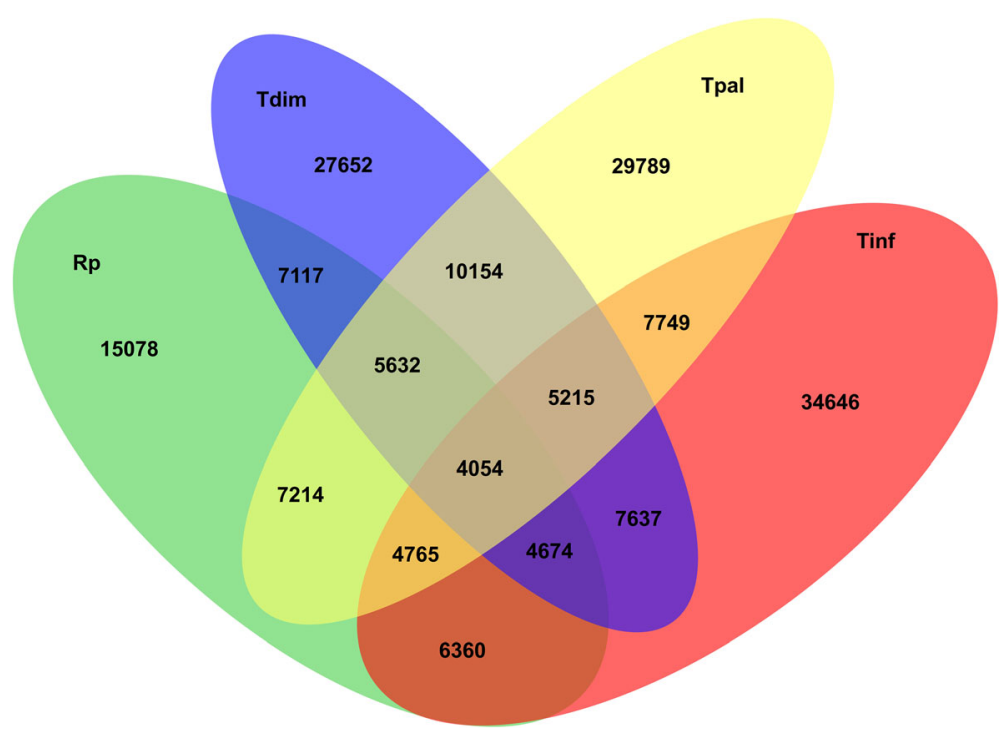

Fig. 1 Putative orthology search within Triatoma and R. prolixus. Bidirectional BLASTX and TBLASTN searches were performed between all four datasets, using the BLAST best reciprocal hit strategy to define putative orthologs. The numbers in the non-overlapping areas correspond to the total number of transcripts in each species. We identified 4054 1:1 orthologs in the four species. Whereas T. pallidipennis and T. dimidiata shared more than 10 thousand 1:1 orthologs, they shared around 7600 with $T$. infestans, recapitulating species phylogeny 
Table 4 GO enrichment in the 3 Triatoma transcriptomes in reference to $R$. prolixus. fisher exact test. FDR $<0.05$

\begin{tabular}{|c|c|c|c|}
\hline GO-ID & Term & Category & Over/Underepresented \\
\hline GO:0005739 & mitochondrion & CC & OVER \\
\hline GO:0005783 & endoplasmic reticulum & CC & OVER \\
\hline GO:0005829 & cytosol & CC & OVER \\
\hline GO:0005840 & ribosome & CC & OVER \\
\hline GO:0043234 & protein complex & $\mathrm{CC}$ & OVER \\
\hline GO:0003729 & mRNA binding & MF & OVER \\
\hline GO:0003735 & structural constituent of ribosome & MF & OVER \\
\hline GO:0008135 & translation factor activity, RNA binding & MF & OVER \\
\hline GO:0019843 & rRNA binding & MF & OVER \\
\hline GO:0030234 & enzyme regulator activity & MF & OVER \\
\hline GO:0051082 & unfolded protein binding & MF & OVER \\
\hline GO:0006091 & generation of precursor metabolites and energy & $\mathrm{BP}$ & OVER \\
\hline GO:0006412 & translation & $\mathrm{BP}$ & OVER \\
\hline GO:0006457 & protein folding & $\mathrm{BP}$ & OVER \\
\hline GO:0006461 & protein complex assembly & $\mathrm{BP}$ & OVER \\
\hline GO:0006605 & protein targeting & $\mathrm{BP}$ & OVER \\
\hline GO:0007005 & mitochondrion organization & $\mathrm{BP}$ & OVER \\
\hline GO:0008219 & cell death & $\mathrm{BP}$ & OVER \\
\hline GO:0022618 & ribonucleoprotein complex assembly & $\mathrm{BP}$ & OVER \\
\hline GO:0042254 & ribosome biogenesis & $\mathrm{BP}$ & OVER \\
\hline GO:0044281 & small molecule metabolic process & $\mathrm{BP}$ & OVER \\
\hline GO:0044403 & symbiosis, encompassing mutualism through parasitism & $\mathrm{BP}$ & OVER \\
\hline GO:0051186 & cofactor metabolic process & $\mathrm{BP}$ & OVER \\
\hline GO:0005886 & plasma membrane & $\mathrm{CC}$ & UNDER \\
\hline GO:0001071 & nucleic acid binding transcription factor activity & MF & UNDER \\
\hline GO:0003677 & DNA binding & MF & UNDER \\
\hline GO:0004871 & signal transducer activity & MF & UNDER \\
\hline GO:0008168 & methyltransferase activity & MF & UNDER \\
\hline GO:0016301 & kinase activity & MF & UNDER \\
\hline GO:0043167 & ion binding & MF & UNDER \\
\hline GO:0007267 & cell-cell signaling & $\mathrm{BP}$ & UNDER \\
\hline GO:0050877 & neurological system process & $\mathrm{BP}$ & UNDER \\
\hline
\end{tabular}

reference. In some cases, significant enrichment (False Discovery Rate, FDR $<0.05$ ) was not congruent with absolute gene number enrichment. To account for confounding effects due to transcriptome expression bias, emphasis was put on those InterPro entries in which absolute transcript counts were greater than in $R$. prolixus in at least one Triatoma species. Enrichment results are summarized in Table 5.

\section{Protease inhibitors and proteases}

A numerical enrichment of transcripts coding for protease inhibitor domains of the pacifastin ( $T$. dimidiata and T. pallidipennis) and cystatin (the three species) families, was identified (Table 5). T. infestans and R. prolixus had nine transcripts/genes encoding pacifastin domains (IPR008037), which corresponded to a statistical enrichment in T. infestans (FDR $3.0^{-2}$ ) (Table 5), although the validation of a pacifastin expansion awaits the availability of $T$. infestans genome sequencing. Pacifastins are a family of protease inhibitors belonging to the MEROPS inhibitor family, involved in the regulation of different proteolytic cascades, including phenoloxidase-dependent melanization [27]. Pacifastin overrepresentation was more remarkable in T. pallidipennis with 17 unique transcripts compared with nine genes in the $R$. prolixus genome $\left(\mathrm{FDR}=6.0^{-5}\right)$. Domain 
Table 5 Fisher's Exact Test for InterPro domain enrichment

\begin{tabular}{|c|c|c|c|c|c|c|c|c|c|c|}
\hline \multirow[t]{2}{*}{ IPR id } & \multirow[t]{2}{*}{ Description } & \multicolumn{4}{|c|}{ \# Genes/transcripts ${ }^{a}$} & \multicolumn{3}{|c|}{ Ref: R. prolixus (FDR) } & \multicolumn{2}{|c|}{ Ref: T. infestans } \\
\hline & & RPRO & TINF & TDIM & TPAL & Ti-Rp & Td-Rp & Tp-Rp & Ti-Td & Ti-Tp \\
\hline IPR005657 & Triabin/Procalin & 46 & 70 & 33 & 32 & $4 \mathrm{E}-32$ & $4 \mathrm{E}-05$ & $2 \mathrm{E}-04$ & $2 \mathrm{E}-04$ & $4 \mathrm{E}-06$ \\
\hline IPR011038 & Calycin-like & 72 & 79 & 36 & 40 & $1 \mathrm{E}-30$ & $2 \mathrm{E}-03$ & $6 \mathrm{E}-04$ & $2 \mathrm{E}-05$ & $4 \mathrm{E}-06$ \\
\hline IPR012674 & Calycin & 86 & 79 & 36 & 40 & $2 \mathrm{E}-27$ & $2 \mathrm{E}-02$ & $1 \mathrm{E}-02$ & $2 \mathrm{E}-05$ & $4 \mathrm{E}-06$ \\
\hline IPR000618 & Insect cuticle protein & 84 & 35 & 27 & 17 & $2 \mathrm{E}-04$ & n.s. & n.s. & n.s. & $4 \mathrm{E}-02$ \\
\hline IPR003286 & RNA-directed DNA polymerase & 0 & 7 & 0 & 0 & $2 \mathrm{E}-04$ & n.s. & n.s. & n.s. & n.s. \\
\hline IPR012336 & Thioredoxin-like fold & 79 & 30 & 38 & 34 & $4 \mathrm{E}-03$ & $2 \mathrm{E}-03$ & n.s. & n.s. & n.s. \\
\hline IPR006170 & Pheromone/general odorant binding protein & 29 & 17 & 13 & 19 & $4 \mathrm{E}-03$ & n.s. & $5 \mathrm{E}-02$ & n.s. & n.s. \\
\hline IPR002557 & Chitin binding domain & 46 & 21 & 10 & 12 & 7E-03 & n.s. & n.s. & n.s. & n.s. \\
\hline IPR008037 & Pacifastin domain & 9 & 9 & 12 & 17 & $3 \mathrm{E}-02$ & 7E-03 & $6 \mathrm{E}-05$ & n.s. & n.s. \\
\hline IPR000010 & Cystatin domain & 1 & 4 & 9 & 6 & n.s & $5 \mathrm{E}-04$ & $5 \mathrm{E}-02$ & n.s. & n.s. \\
\hline IPR020849 & Small GTPase superfamily, Ras type & 15 & 3 & 6 & 19 & n.s & n.s. & $2 \mathrm{E}-04$ & n.s. & n.s. \\
\hline IPR000217 & Tubulin & 19 & 6 & 15 & 13 & n.s & $2.3 \mathrm{E}-02$ & n.s. & n.s. & n.s. \\
\hline IPR001254 & Serine proteases, trypsin domain & 89 & 1 & 34 & 31 & n.s & n.s & n.s & $1 \mathrm{E}-04$ & $2.0 \mathrm{E}-03$ \\
\hline
\end{tabular}

${ }^{a}$ Total number of InterPro annotated genes/transcripts: T. dimidiata: 1884; T. infestans: 1419; T. pallidipennis: 2110; R. prolixus: 10,407

architecture analysis revealed novel domain configurations in $T$. pallidipennis absent in $R$. prolixus predicted proteome such as a pacifastin with von Willebrand factor, type C (IPR001007) and D domains (IPR001846), as well as a membrane-anchored two pacifastin-repeat coding transcript, and a six pacifastin domain transcript encoding a putative secreted product (Fig. 2). A membrane-anchored pacifastin containing a Kazal-type protease inhibitor domain (IPR002350) present in $R$. prolixus was not found in the three - Triatoma species (Fig. 2). A detailed characterization of pacifastin domain-encoding transcripts in the nonredundant datasets is in Additional file 1.
Transcripts encoding for InterPro domains belonging to protease inhibitors other than pacifastins were also more abundant. Cystatins are important regulators of cathepsin-mediated intracellular proteolysis [28, 29]. There is only one cystatin domain-type (IPR000010) protease inhibitor gene in $R$. prolixus, but cystatins were more abundant in T. dimidiata $(n=9), T$. pallidipennis $(n=6)$, and T. infestans $(n=4)$.

Serine-type protease gene expansions are common in hematophagous arthropods $[14,30]$. Enrichment of trypsin-domain serine proteases (IPR001254) was not found in any of the three Triatoma species. However, serine proteases were numerically and statistically

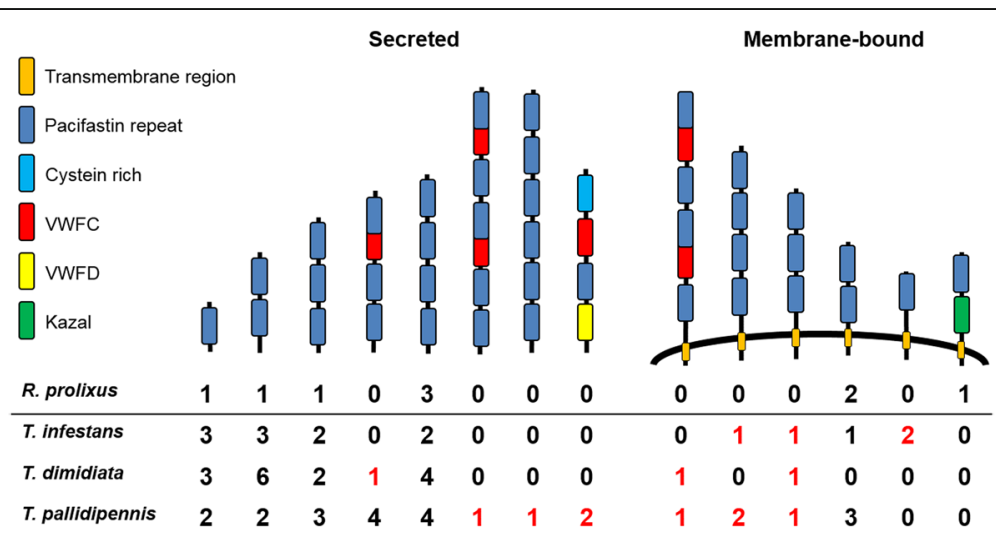

Fig. 2 Structural characterization of the pacifastin family in the three - Triatoma species and R. prolixus. Based on InterPro annotations, transcripts of the non-redundant dataset (including singletons) were classified according to the presence of signal peptide, transmembrane regions and other InterPro domains. Absolute numbers for each type and species are shown in the matrix. Apart from the increase in gene number, particularly observed in T. pallidipennis and T. dimidiata (Table 4), we observed divergence and evolutionary innovation in secreted and membrane-bound pacifastins (red numbers). WWFC, von Willebrand Factor C domain; WFD, von Willebrand Factor D domain. A detailed description of the pacifastin domain coding transcripts in Triatoma is found in Additional file 1 
depleted in $T$. infestans, regarding $T$. dimidiata $(\mathrm{FDR}=$ $\left.1.0^{-4}\right)$ and T. pallidipennis $\left(\mathrm{FDR}=2 \cdot 0^{-3}\right)$.

\section{Calycins}

Calycins belong to a large family of extracellular proteins involved in a variety of functions, including binding of lipophilic compounds. A structurally related sub-family, the triabin/procalin family is an important component of hematophagous insects, including Triatoma, and function as anti-hemostatic factors [20,31]. Transcripts containing the calicyn fold (IPR012674) were statistically enriched in the three - Triatoma species (Table 5). In particular, the calycin-like fold were enriched in T. infestans $(n=79)$, compared to $R$. prolixus $(n=72)\left(\mathrm{FDR}=1.0^{-30}\right)$. This calycin enrichment can be attributed to a significant triabin/procalin (IPR005657) expansion in the three species, but again higher in $T$. infestans ( $n=70$ versus 40 in R. prolixus. FDR $=4 \cdot 0^{-32}$ ). As expected, the calycin fold was also enriched in $T$. infestans over T. pallidipennis $(n=40$, $\left.\mathrm{FDR}=4 \cdot 0^{-6}\right)$ and $T$. dimidiata $\left(n=36, \quad \mathrm{FDR}=2 \cdot 0^{-5}\right)$. Calycins are classified according to the $\mathrm{GO}$ biological process "symbiosis, encompassing mutualism through parasitism" (GO:0044403), explaining the corresponding GO term enrichment in Triatoma sp., particularly in T. infestans (Table 5).

Our transcriptome datasets are consistent with the high structural complexity of the calycin family. To characterize the calycin types and their functions that were expanded in the three - Triatoma species, we followed a pair-wise comparison clustering approach, which included previously identified metazoan calycins along with those identified in our datasets. Through CLANS clustering analysis [32], we identified 11 major clusters in our Triatoma transcriptomes that corresponded to procalins (clade I), triatins (clade II), salivary lipocalins (clade IV), triafestins (clade V), collagen-induced platelet aggregation calycins (clade VI), and lipocalins/palidipinlike proteins (clade VII) [31]. However, we did not identify any procalin orthologs in $T$. infestans, and triabins - clade III calicyns in our Triatoma transcriptomes (Fig. 3).

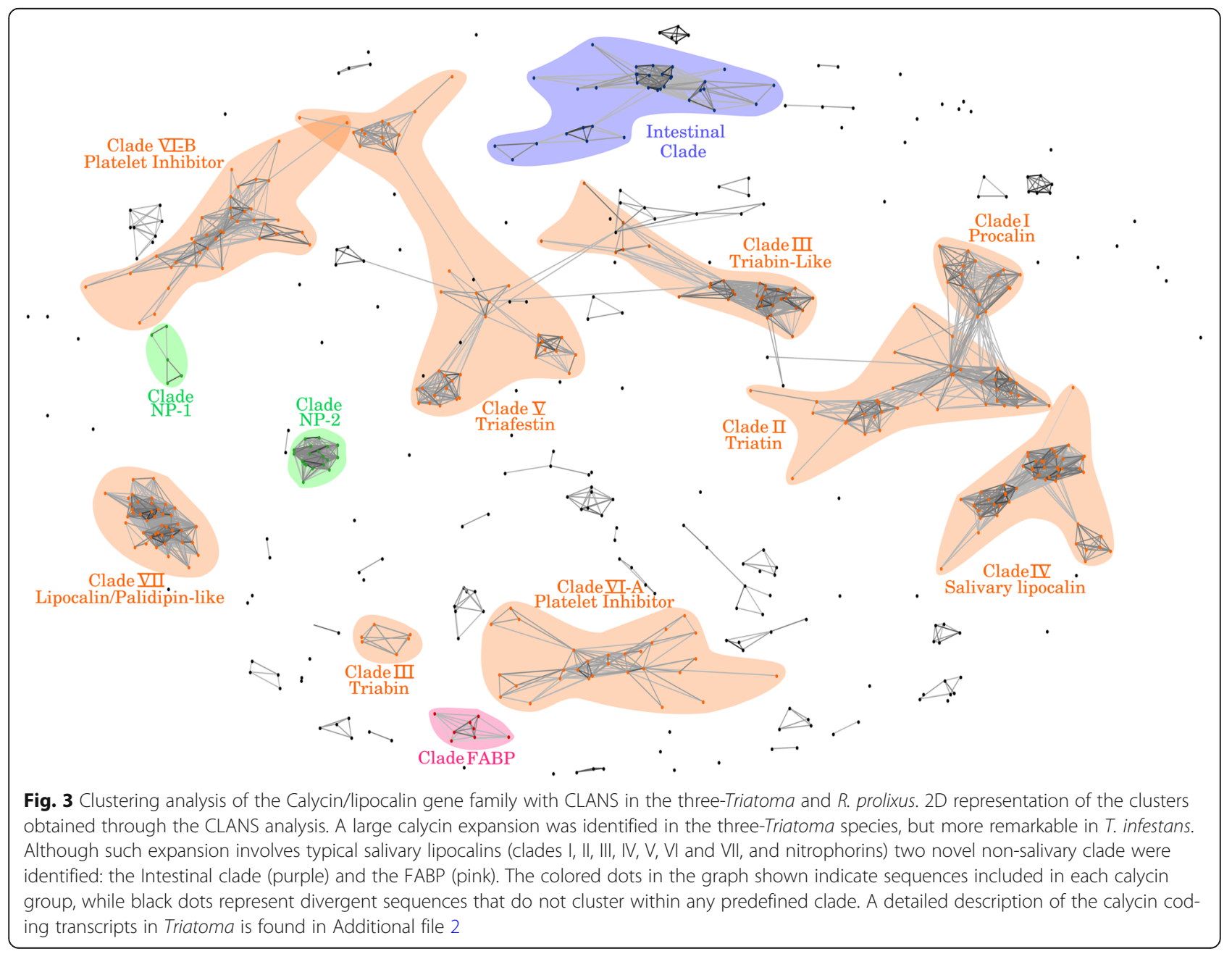


In addition, we found several $T$. infestans, $T$. dimidiata and $T$. pallidipennis contigs sharing high sequence identity with nitrophorins, salivary heme-containing proteins, which participate in nitric oxide (NO) transport and storage [33]. These transcripts clustered with recently classified $R$. prolixus nitrophorins (RPRC000537 and RPRC000163) (green clusters, Fig. 3) [14], but not with the $R$. prolixus nitrophorins NP1-NP4.

One interesting finding from the clustering analysis was the identification of two clusters that lacked salivary calycins in the three -Triatoma species. In one of them, many sequences clustered with previously identified lipocalins in the intestinal transcriptome of $R$. prolixus [34] (purple clade, Fig. 3), referred here after as the intestinal clade. In the other group, sequences clustered with two $R$. prolixus sequences and fatty-acid binding proteins (FABP) highly conserved in insects and vertebrates (pink clade, Fig. 3). A detailed description of the three - Triatoma calicyn family clusters is provided in Additional file 2.

\section{Transposable elements}

Nine unique transcripts containing the RNA-directed DNA polymerase domain (IPR003286) were found in $T$. infestans, but none in $R$. prolixus and the other Triatoma datasets. A detailed mapping against the RepBase database [35] revealed a marked increase in the absolute numbers of transcriptionally active Long Terminal Repeat (LTR), non-LTR retrotransposons, and DNA transposable elements in $T$. infestans $(n=106)$, compared with $T$. dimidiata $(n=13)$ and T. pallidipennis $(n=23)$ (Fig. 4a, Additional file 3). The majority of $T$. infestans TEs were non-LTRs (46\%), mainly CR1, Jockey, Nimb and Loa-type; followed by DNA TEs (36\%), mainly

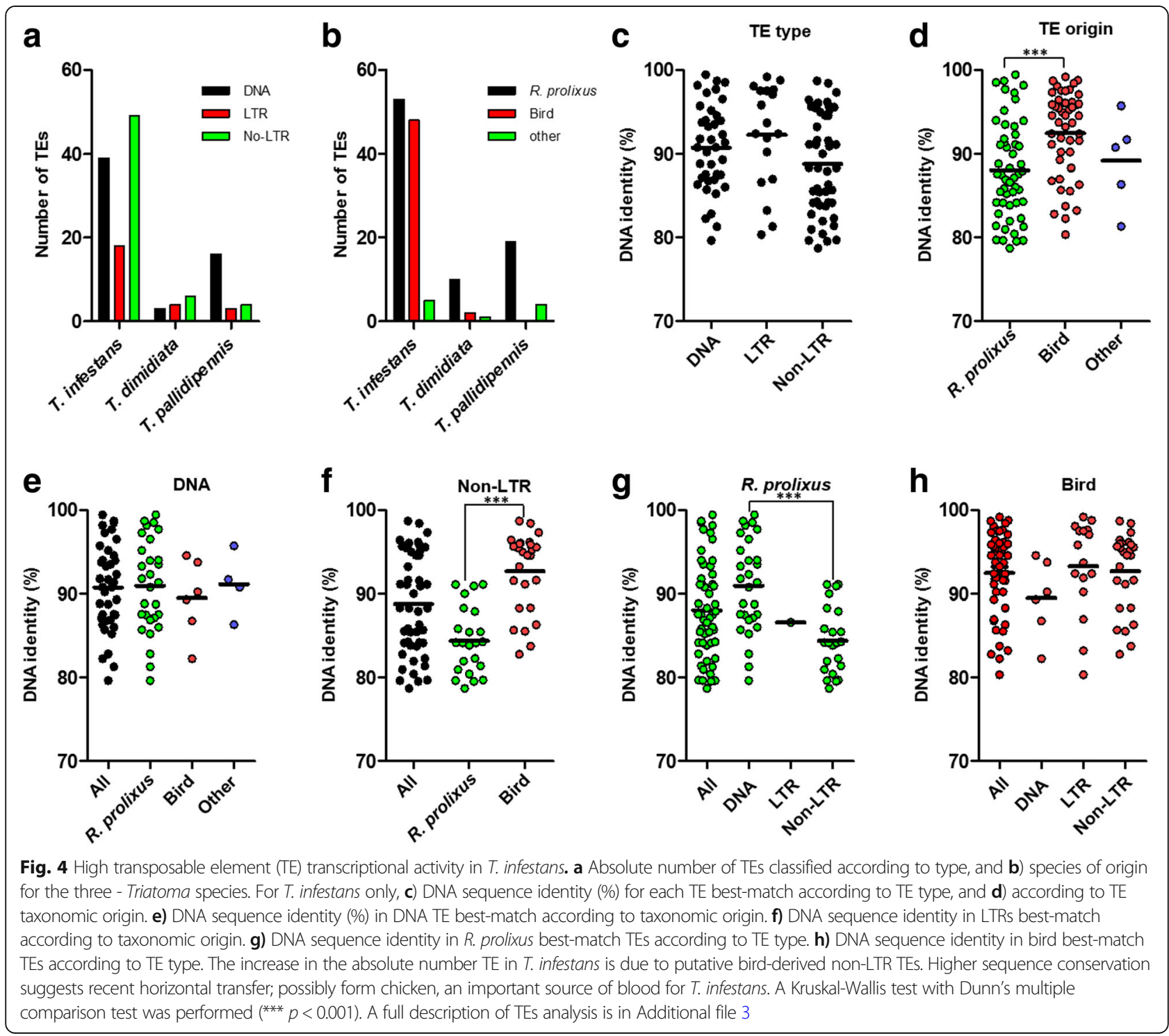


Mariner-like. There was at least a five-fold increase in the number of LTRs in T. infestans (16\% of all TEs), mainly ERV-type. In T. pallidipennis, the majority were DNA TEs (69\%), being Mariner-like the most common (Additional file 3).

Although half of TEs identified in T. infestans best matched with $R$. prolixus TEs, a large proportion (45\%) best matched to bird TEs (Fig. $4 \mathrm{~b}$ and $\mathrm{d}$ ). These sequences were mainly CR1-like non-LTRs and ERV-like LTRs (Additional file 3). Nucleotide sequence identity of T. infestans non-LTRs (CR1-like) with their corresponding bird best match was significantly higher than those best-matching $R$. prolixus non-LTRs (Jockey, Nimb and Loa) (Fig. 4d and f) and DNA TEs (Fig. 4g) $(p<0.001)$ (Additional file 3).

\section{Olfaction gene families}

A statistically significant enrichment of odorant binding proteins (OBPs) (IPR006170) was found in T. pallidipennis and T. infestans (Table 5). Because of their role in feeding preference and mating and other behavioral traits [36], we further searched for chemoreceptorcoding transcripts. We identified between 106 and 123 chemosensory genes distributed belonging to the most representative gene families among the three transcriptomes: odorant receptors (PF02949); ionotropic receptors (PF00060); gustatory receptors (PF08395); chemosensory proteins (PF03392); SNMP/CD36 (PF01130); mechanoreceptors (PF02949); and pickpocket receptors (PF00858) (Additional file 4). Taking into account putative orthologs (BRH between Triatoma and $R$. prolixus), OBPs and CSPs were the most abundant families. As for pacifastins in T. infestans, validation of the OBP family expansion awaits the availability of the full genome sequence of the corresponding triatomine.

\section{Other protein families}

An additional number of protein families/domains were enriched in the three -Triatoma species or in a particular species (Table 5). The number of Ras-type, small-GTPase superfamily (IPR020849) encoding transcripts was increased only in T. pallidipennis $(n=19$ vs. 14 in $\mathrm{R}$. prolixus; $\mathrm{FDR}=2.0^{-4}$ ). Other protein families were enriched only in statistical terms. The thioredoxin-like domain (IPR012336) was enriched in the three species; however, absolute numbers were lower than in $R$. prolixus. In $T$. dimidiata, there were 15 tubulin (IPR000217) transcripts compated to 19 in $R$. prolixus $\left(\mathrm{FDR}=2.3^{-2}\right)$. Finally, statistical enrichment regarding $R$. prolixus of insect cuticle protein (IPR000618) $\left(\mathrm{FDR}=2.0^{-4}\right)$ and chitin-binding domains (IPR002557) $\left(\mathrm{FDR}=7.0^{-3}\right)$ was found in $T$. infestans, which might be implicated in cuticle formation, melanization and perimicrovilliar membrane formation [37-39]. As previously stated, validation of a gene expansion in these gene families awaits the availability of the full genome sequence of these triatomines.

\section{Protein divergence according to functional classes}

To gain insight into protein family evolution in triatomines, we assessed protein sequence divergence according to GO class as a relative measure of evolutionary rate among each Triatoma and $R$. prolixus 1:1 orthologs. To compare protein sequence conservation within Triatoma species, $\mathrm{Z}$ values (number of standard deviations from the mean \% identity) were used. The majority of GO classes were distributed around $\mathrm{Z}=0$ (i.e. average divergence), but few classes had negative $Z$ values (more conserved than the average), while fewer classes had positive $Z$ values (more divergent than the average) (Fig. 5a).

$\mathrm{Z}$ values according to $\mathrm{GO}$ biological process and molecular function were clustered, based on their Pearson's correlation by species and represented in a pseudocolor heatmap (Fig. 5b-c. Additional file 5). Putative ortholog pairs belonging to core biological process such as "cell signaling", "cellular component biogenesis and organization", "autophagy", "morphogenesis" and "cellular responses to stimulus" were more conserved among species (Fig. 5b, negative $\mathrm{Z}$ scores in blue), although only "cellular response to stimulus" (GO:0051716) and "cellular component biogenesis" (GO:0044085) were statistically significant (Kruskal-Wallis one Way ANOVA. Dunn's correction for multiple testing, $p<0.05$ ). A high proportion of orthologs belonging to this GO term participate in protein synthesis and different components of the translation machinery and cell signaling. In contrast, orthologs annotated as "single-organism metabolic processes" (GO:0044710) were significantly less conserved in the three - Triatoma species (Kruskal-Wallis one Way ANOVA. Dunn's correction for multiple testing, $p<0.05$ ). Only in T. infestans, orthologs annotated as "interspecies interaction between organisms" (GO:0044419) were significantly less conserved (Fig. 5b. Positive Z scores in yellow. Additional file 5). Calycins belong to this term, indicating that in addition their numerical expansion; they are evolving at a fast rate in T. infestans.

Consistently, the same analysis based on "molecular function" and "cellular component" GO's revealed high protein coding sequence conservation in the three Triatoma species for orthologs involved in protein synthesis and nucleic acid or nucleoside binding. In particular, orthologs belonging to "structural constituent of ribosome" (GO:0003735), "heterocyclic compound binding" (GO:1901363), and "localization in the ribonucleoprotein complex" (GO:1990904) were significantly conserved at the protein sequence level. In contrast, 


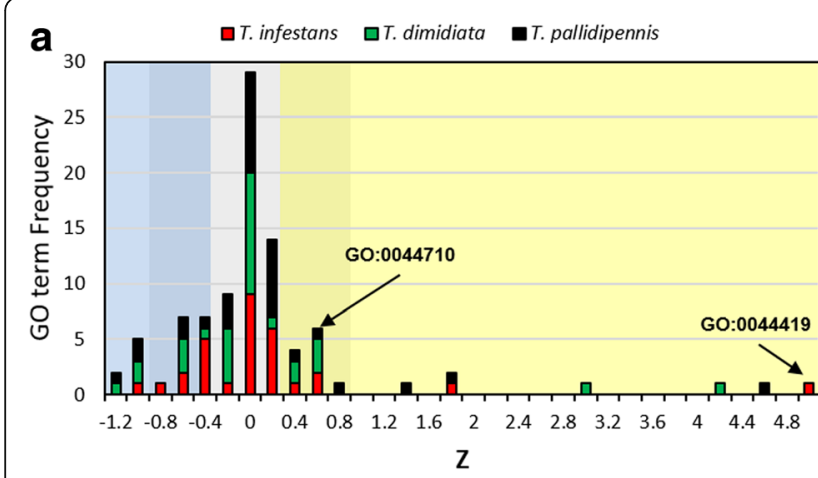

C

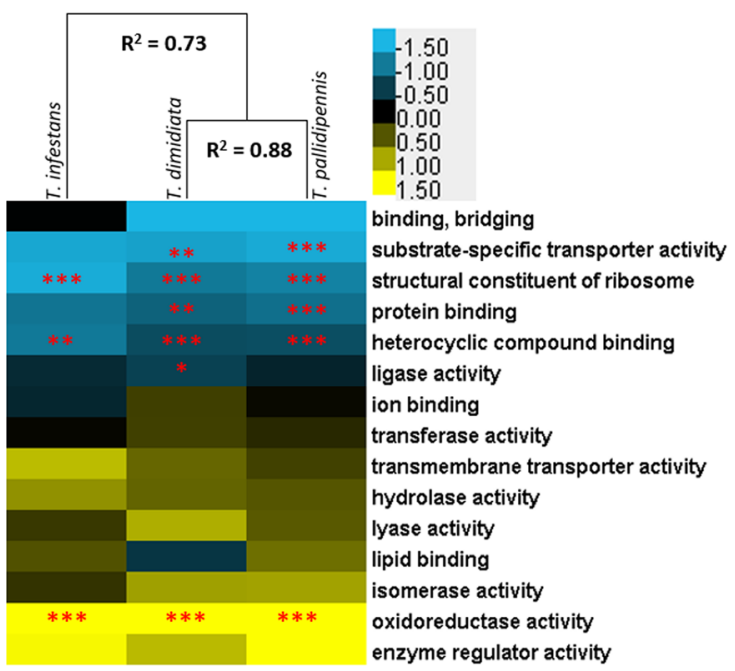

b

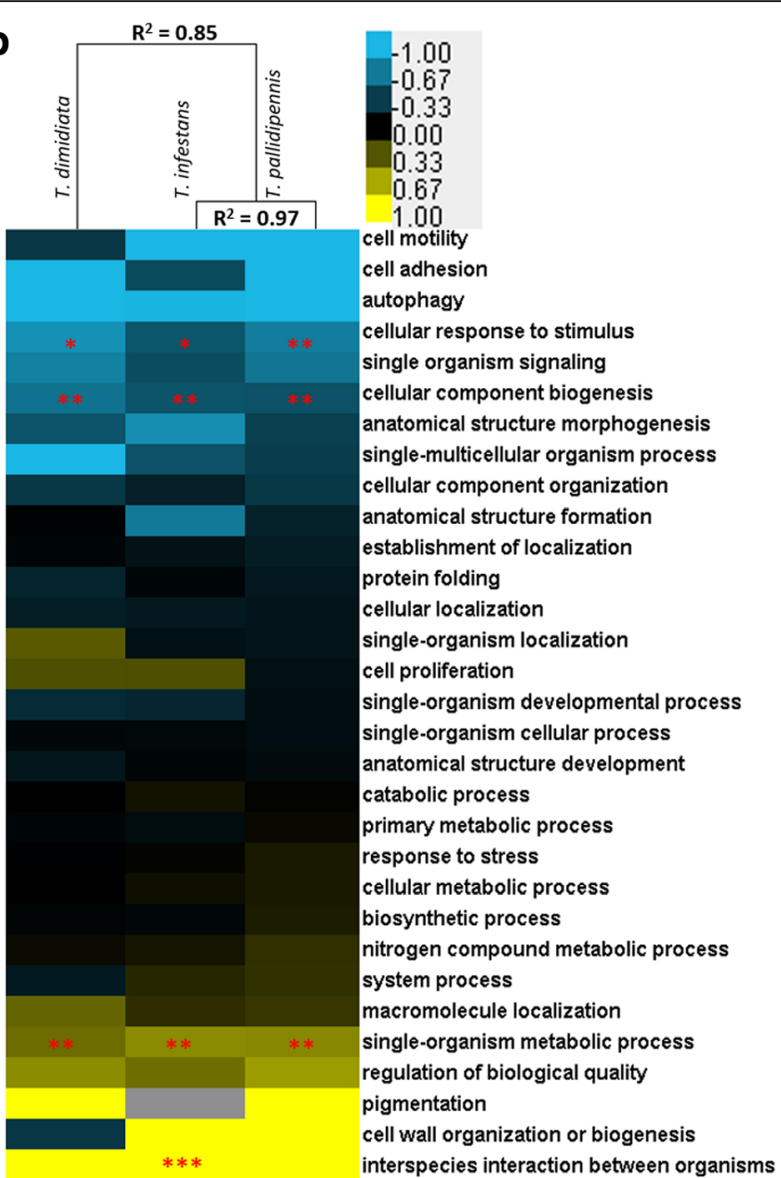

Fig. 5 Structural divergence between the three - Triatoma 1:1 orthologs (BLAST best reciprocal hit to R. prolixus). a Distribution of GO (biological process) term frequency ( $y$ axis) according to $Z$ value (the number of standard deviations from the median mean), as a measure of protein divergence ( $x$ axis). Negative $Z$ values indicate higher conservation, whereas positive $Z$ values indicate higher divergence. For instance, GO:0044710 ("single organism metabolic process") has the same Z value in the three species, but is less divergent than GO:0044419 ("interspecies interaction between organisms"), with different although high Z values in the three species. b Biological process Z values for level 3 Gene ontology terms GO's (rows). The Z value was numerically ordered according to T. pallidipennis and hierarchically clustered (Pearson's correlation) according to species (columns). Blue tones reflect higher protein sequence \% identity (i.e. more conserved), whereas yellow tones reflect lower \% identity (more diverged). Significant higher protein sequence divergence were found in "single organism metabolic processes", "interspecies interaction between organisms", and oxidoreductase activity. c Molecular function Z values. Gene ontology terms level 3 GO's. Kruskal-Wallis one Way ANOVA. Dunn's correction for multiple testing. ${ }^{* * *} p<0.001 ;{ }^{* *} p<0.01$. Non statistical significance in some conserved and divergent GO classes was probably because the lack of power due to low numbers of genes in those classes. Data corresponding to this figure, including \% identity mean for each GO term and cellular compartment terms is in Additional file 5

significant higher protein sequence divergence was observed in "oxidoreductases" (GO:0016491) in the three species (Fig. 5c, Additional file 5). Many of these oxidoreductases are involved in respiration and other metabolic processes, which is in agreement with the observed divergence in "single-organism metabolic process" (GO:0044710) (Fig. 5a-b). In addition, "enzyme regulator activity" (GO:0030234) was the most divergent molecular function term, although this finding was not statistically significant, probably due to small sample size. Pacifastins and other protease inhibitors belong to this category; suggesting that proteolysis regulation trait in Triatoma may be evolving at a high rate (Additional file 5).
Hierarchical clustering of protein sequence conservation ( $\mathrm{Z}$ scores) according to biological process (Fig. $5 \mathrm{~b}$ ) and cellular component (Additional file 5) did not correlate with the corresponding Triatoma phylogenetic relationships, in which T. pallidipennis and T. dimidiata are more closely related than with $T$. infestans. However, clustering according to molecular function did recapitulate the established phylogeny. (Fig. 5c).

To identify genes/functions that show higher protein sequence divergence, we asked if the most divergent ortholog pairs were enriched in more specific GO terms. One-to-one ortholog pairs were ranked according to protein pairwise identity (\% identity with BLASTx) and the whole ranked dataset was subdivided into equal 
terciles or groups (Additional file 6). Using the most conserved group as reference (Additional file 6, blue), GO enrichment analysis (Fisher's Exact test) confirmed that orthologs involved in cellular metabolism, particularly in carbohydrate metabolism and oxidative phosphorylation were enriched in the most divergent group (Additional files 6 and 7. yellow). This included many oxidoreductases participating in the respiratory chain complexes (Additional file 7). It also confirmed high protein divergence in the calycin family in T. infestans ("evasion or tolerance of host defense response", GO: 0030682), and "peptidase inhibitor activity", GO: 0030414) in T. pallidipennis. Importantly, biological processes such as carbohydrate, ketone and lipid metabolism were enriched in the most divergent group, suggesting that the whole energy generation and storage pathway may be evolving at higher rates. Additionally, "serine-type endopeptidase activity" (GO:0004252) was enriched in the most divergent group only in $T$. dimidiata and T. pallidipennis, indicating that, in addition to a numerical expansion (Table 5), serine proteases may be evolving at a high rate in these species regarding $T$. infestans.

\section{Reconstruction of the carbohydrate metabolic pathways}

The 1:1 Triatoma- $R$. prolixus orthologs identified in the RproC3.2_mapped dataset involved in glycolysis/gluconeogenesis and pentose phosphate pathway (PPP) [14], as well as in threalose, glycogen and triglyceride metabolism were identified and labeled according to their protein divergence groups (Fig. 6. Additional file 8). Among $43 R$. prolixus genes, orthologs for 36 were found in the three - Triatoma species, and the majority of these belonged to the intermediate or most conserved group. Only five orthologs involved in carbohydrate metabolism with coverage higher than $40 \%$ belonged to the most divergent group. A putative phosphoglucomutase 1 (Pgm1) ortholog, involved in glycolysis and glycogen synthesis was found in T. pallidipennis, but absent in $T$. dimidiata and T. infestans (Fig. 6). The T. pallidipennis ortholog for pyruvate kinase $(P y k)$ of the glycolytic pathway, fructose-1,6-bisphosphatase $(F b p)$ involved in gluconeogenesis was identified in the three - Triatoma species. Interestingly, only $F b p$ in $T$. dimidiata and $T$. pallidipennis belonged to the most divergent group, whereas the corresponding $T$. infestans ortholog belonged to the most conserved group (Fig. 6).

Among six $R$. prolixus genes encoding for PPP enzymes, only glucose-6-phosphate dehydrogenase $(G 6 p d)$ was not found in the three - Triatoma species. A putative phosphoglucomutase/phosphopentomutase 2 (Pgm2), involved in purine and pyrimidine biosynthesis was found in $T$. pallidipennis and $T$. infestans, but only in the later belonged to the most divergent group (Fig. 6). The T. pallidipennis transaldolase (Tald) orthologs, linking the PPP to glycolysis, also belonged to the most diverse group (Fig. 6 . Additional file 8).

\section{Divergence in oxidative phosphorylation}

ATP production by oxidative phosphorylation is mediated by at least five mitochondrial multi-subunit complexes involved in electron transport, generation of a $\mathrm{H}+$ gradient across the mitochondrial membrane and coupling such gradient to ATP synthesis. As for metabolic pathways, most of nuclear and all mitochondrial gene orthologs coding for the NADH dehydrogenase complex (I), succinate dehydrogenase complex (II), cytochrome B complex (III), cytochrome c oxidase complex (IV) and ATP synthase complex (V) were identified in $R$. prolixus and the three - Triatoma species. A significant proportion of subunits of complex I, IV and V belonged to the most divergent group (Additional file 9).

\section{NADH dehydrogenase complex (I)}

Complex I catalyzes the electron transfer from $\mathrm{NADH}$ to ubiquinone, as well as proton translocation into the inter-membrane space. The eukaryotic complex I core is composed by 14 subunits (seven encoded in the mitochondrial genome), an $\alpha$ subcomplex composed by 13 nuclear-encoded subunits, and a $\beta$ subcomplex composed by 11 nuclearencoded subunits. We identified 11 core subunits, 10 $\alpha$ subcomplex subunits, and all $\beta$ subcomplex subunits in the three - Triatoma species and $R$. prolixus. All the mitochondria-encoded subunits, two and seven subunits of the $\alpha$ and $\beta$ subcomplex, respectively belonged to the most divergent group

(Additional file 9).

\section{Succinate dehydrogenase complex (II)}

Complex II participates in the TCA cycle and contributes as an electron donor to the ubiquinone pool. We identified all four subunits of complex II in the three Triatoma and $R$. prolixus. Only SdhD were in the most divergent group (Additional file 9).

\section{Cytochrome B complex (III)}

Complex III transfers electrons to cytochrome c from the ubiquinone pool and contributes to the proton gradient by translocating four $\mathrm{H}+$. Nine of the 11 subunits of complex III were identified in the four species. Only two, QCR2 and QCR7 were in the most divergent group (Additional file 9).

\section{Cytochrome oxidase C complex (IV)}

In Complex IV, electrons in cytochrome c (complex III) are transferred to oxygen to form water, linked to the 


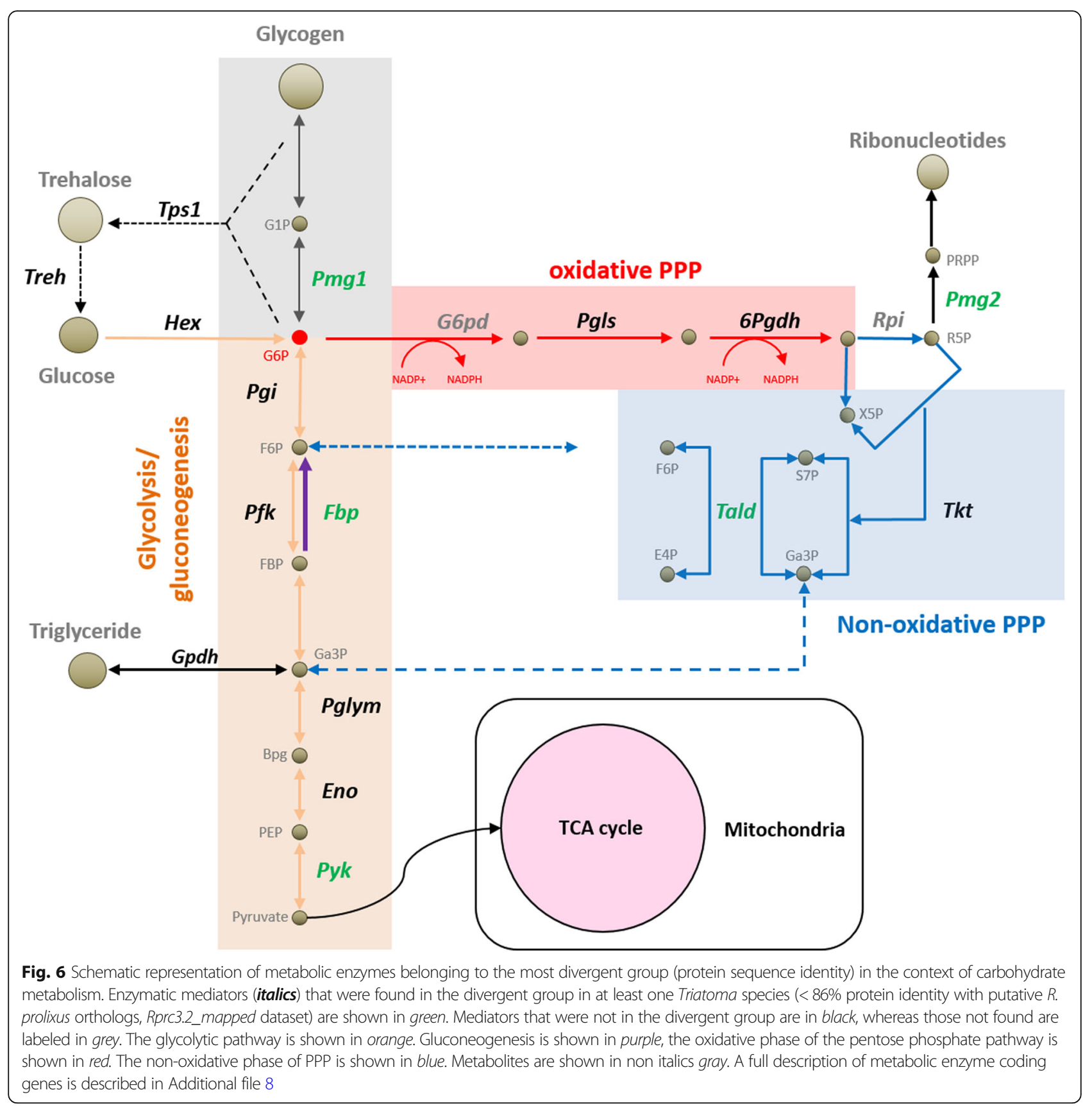

translocation of additional protons. Complex IV is composed by 20 subunits. Cox $1, \operatorname{Cox} 2$ and Cox3 are mitochondria-encoded. We identified 12 subunit orthologs, five of which were in the most divergent group in the three - Triatoma species (Additional file 9).

\section{ATP synthase complex (V)}

The ATP synthase couples the electrochemical proton gradient with the generation of ATP. It is composed of a $F_{O}$ subcomplex, which constitutes the proton pore embedded in the inner mitochondrial membrane, and a $F_{1}$ subcomplex, which mediates ATP synthesis.
The $F_{O}$ subcomplex is composed of at least eight subunits, two of which are mitochondria-encoded (ATP6 and ATP8). The $F_{1}$ subcomplex is composed of five nuclear-encoded subunits $(\alpha, \beta, \gamma, \delta$ and $\varepsilon$ ). Orthologs for the eight $F_{O}$ subunits and all $F_{1}$ were identified in the three - Triatoma and $R$. prolixus. All the components of the $F_{O}$ subunit, but subunit $C$ (pore-forming subunit), belonged to the most divergent group, while all $F_{1}$ subunits were in the most or intermediately conserved groups (Additional file 9).

Other enzymes beyond complexes I-V participate in energy/redox metabolism, such as mitochondrial 
Glycerol 3-phosphate dehydrogenase (mG3P) and Proline dehydrogenase (ProDH), which may play an important role in insect vectors physiology [40, 41]. The corresponding mG3P orhtolog was found in $R$. prolixus and the three triatomas, although only in $T$. dimidiata it belonged to the most divergent group. In the case of ProDH, no orthologs were found in T. dimidiata, but singleton BRH's were found in T. infestans in most divergent group, and in $T$. pallidipennis in the average conserved group (Additional file 9).

We further tested if the genes coding for the oxidative phosphorylation complexes subunit orthologs were under positive (adaptive) selection in 23 orthologs of the NADH dehydrogenase complex (I), six of the cytochrome B complex (III), 12 of the cytochrome C complex (IV) and 13 of the ATP synthase complex (V) using MEGA7 (Kumar, et al. 2016). No evidence of positive selection $(\mathrm{dN}>\mathrm{dS})$ was found using a codon-based Z-test of positive selection $(p>0.05)$. Furthermore, we used the HyPhy package to estimate selection per codon, based on the difference of non-synonymous $(\mathrm{dN})$ and synonymous (dS) substitutions maximum likelihood [42]. Positive normalized $\mathrm{dN}-\mathrm{dS}$ values indicate higher likelihood that the codon is under positive selection (Fig. 7). Most codons presented negative normalized $\mathrm{dN}-\mathrm{dS}$ values, which is consistent with negative selection. We detected positive normalized $\mathrm{dN}$ - $\mathrm{dS}$ values in many codons for several orthologs, suggesting positive selection, although these were not statistically significant. However, comparison of normalized $\mathrm{dN}-\mathrm{dS}$ distribution of each ortholog revealed a significant bias towards positive normalized $\mathrm{dN}-\mathrm{dS}$ values in six NADH dehydrogenase $\beta$ subcomplex subunits, two $\alpha$ subcomplex subunits and all mitochondrial genome-encoded complex I core subunits (Fig. 7a). For complex III, significant differences in Cyt B and QCR2 dN-dS distribution was found (Fig. 7b). In contrast to mitochondrial-encoded subunits, several nuclear encoded subunits of cytochrome c oxidase complex, particularly $\mathrm{COX}_{\mathrm{A}}$, showed biased $\mathrm{dN}$-dS distribution towards positive values (Fig. 7c).

In the case of the ATP synthase complex, consistent with the segregation of protein sequence divergence between the $F_{1}$ and $F_{O}$ subunits (Additional file 9), a remarkable segregation of $\mathrm{dN}-\mathrm{dS}$ distribution was also detected. All components of the catalytic $F_{1}$ subcomplex showed strong bias towards negative $\mathrm{dN}-\mathrm{dS}$ values, whereas all $F_{O}$ subcomplex subunits were significantly biased toward positive values (Fig. 7d).

\section{Discussion}

We conducted a transcriptomic analysis of normalized cDNA libraries that cover the entire life cycle of three triatomine species relevant for Chagas disease transmission from Mexico to Argentina, T. pallidipennis, $T$ dimidiata and T. infestans. To get insights into the triatomine biology, we followed a "data driven" research approach (gene content, protein family architecture, protein sequence divergence and positive selection inference) towards the identification of genetic signatures that could suggest adaptations to their particular and diverse life styles. From this approach, we expected to generate novel hypothesis regarding triatomine biology, rather than providing exhaustive characterization and hypothesis testing of genes involved in different biological processes.

Almost two-thirds of our de novo assembled datasets map to the $R$. prolixus genome and proteome, and three quarters of the $R$. prolixus proteome had a homologous match in the three - Triatoma datasets. Despite the relatively low throughput of the 454 sequencing technology compared with other sequencing technologies, library normalization contributed to the high coverage of the core eukaryotic genome (CEG) [23] and the core arthropod single-copy ortholog dataset (BUSCO) [24] (Table 2). Thus, the coverage estimation in our datasets sufficed for a meaningful analysis of the transcriptome and genome gene content comparisons. Despite normalization, genes expressed at very low levels or restricted to few cells could contribute to the incompleteness of the datasets. Gene family contraction and expansions are common during speciation and adaptation to ecological niches [43]; however we are aware that not all gene family expansions are adaptive [44]. Therefore, we focused on the analysis of gene family enrichment in absolute terms, which accurately reflect true gene expansions, and we suggest caution in the interpretation of those cases with statistical evidence, but no absolute numeric gene increase data. Deep transcriptome sequencing analysis of less abundant cell types, as well as full genome sequencing of the corresponding Triatoma species will be required for definitive genome content comparisons among reduviids.

InterPro annotation enrichment in the triatomines was assessed using $R$. prolixus as reference (Table 5). Remarkably, two types of protease inhibitors (PI) were numerically enriched in the triatomines: pacifastins and cystatins. Pacifastins were expanded at least in T. dimidiata and T. pallidipennis. Pacifastins were described in crustaceans as serine protease inhibitors that regulate the phenoloxidase cascade [45]. They are widely distributed in arthropods and almost all insect orders [27, 46]. Pacifastins were described in T. infestans sialotranscriptome [47], and an up-regulated pacifastin upon blood meal and bacterial challenge, was described in T. infestans fat body [48], suggesting that pacifastins may be important regulators of hematophagy and immune response in triatomines. 


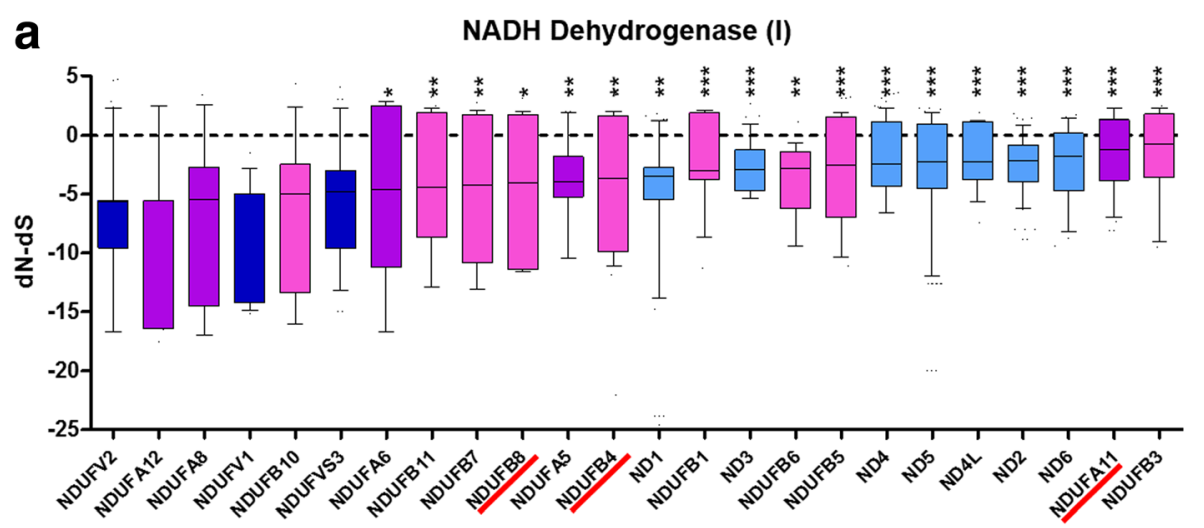

b

C

Cytochrome C oxidase (IV)
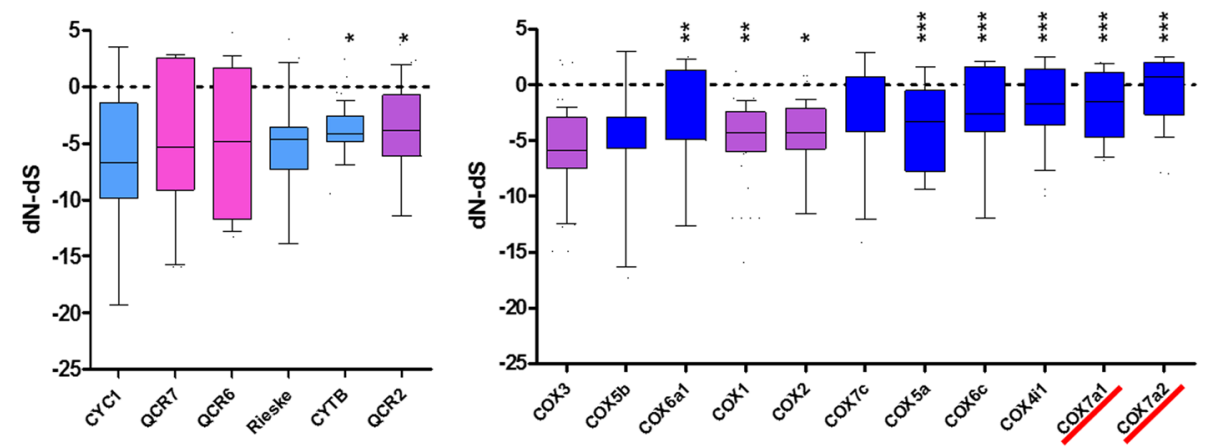

d

\section{ATP synthase (V)}

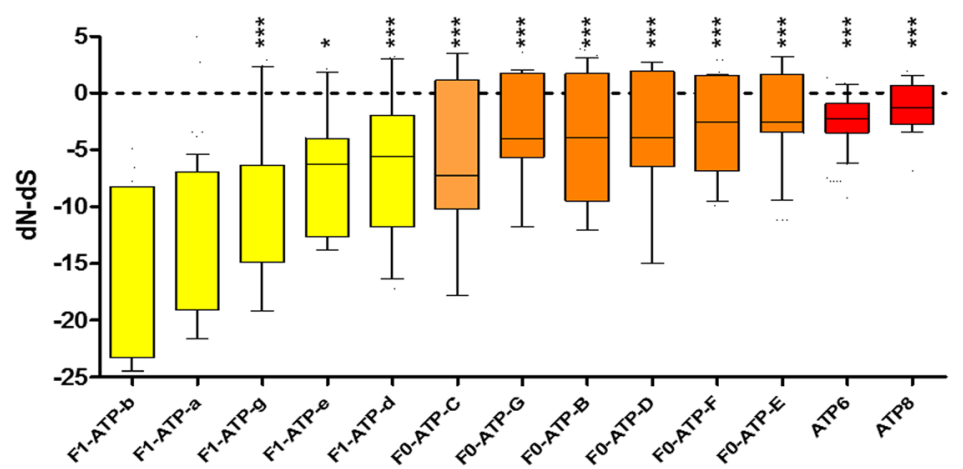

Fig. 7 Distribution of normalized dN-dS in reduviid oxidative phosphorylation complex subunits. Codon-by-codon Maximum Likelihood analysis was calculated for each ortholog using the HyPhy package [42]. The normalized dN-dS for each variant position was plotted according to increasing median value from left to right. Positive values indicate likelihood of selection. a Complex I. Core mitochondrial subunits (light blue), core nuclear-encoded subunits (dark blue), accessory A subunits (purple), accessory B subunits (pink); b Complex III. Respiratory subunits (light blue), core subunit (purple), low molecular weight subunits (pink); c Complex IV. Mitochondria-encoded subunits (purple); and d) ATPase complex. F1 ATPase subunits (yellow), Nuclear-encoded F0-ATPase subunits (orange) and mitochondria-encoded F0 subunits (red). Significant differences (one-way ANOVA test corrected for multiple comparison) in $\mathrm{dN}$-dS distribution are shown under black bar. Red arrows indicate subunits participate in the

oligomerization of respiratory supercomplexes in mammals [77]. A full description of coding genes involved in oxidative phosphorylation is described in Additional file 9

In the three - Triatoma species, we found no evidence of the Kazal domain containing-pacifastin present in $R$. prolixus, but its absence in Triatoma sp. cannot be ruled out. Although, our triatomine pacifastin architecture reconstruction may be incomplete due to the possibility of non-full length transcripts, the identification of novel domain configurations (Cystein-rich, VWFC and VWFD domains), particularly in membrane bound pacifastins (Fig. 2, Additional file 1), indicates evolutionary innovation in this protein family and supports the current view of adaptive diversification in the pacifastin family [46]. 
Cystatins belong to a large and widespread family of cysteine proteases (i.e. papain, cathepsins) inhibitors in plants, prokaryotes and animals [29]. Cystatins also play an antihemostatic role during blood meal in ticks $[49,50]$; but they appear to be absent in the Triatoma sialotranscriptomes described so far [15-17, 31, 47, 51]. Tigutcystatin, a secreted midgut cystatin, may be involved in blood digestion and host - T. cruzi interaction in T. infestans [52], thus the cystatin expansion we documented in T. pallidipennis and T. dimidiata deserves further study to elucidate its biological significance. Taken together, gene expansions in two protein-inhibitor unrelated families (Table 5), with different cellular localization, substrate specificities and higher evolutionary rates (Fig. 5, Additional file 5), suggest high evolutionary dynamics in proteolysis regulation in triatomines. This includes, but is not limited to, adaptations in hematophagy, immune response and parasite-vector interactions.

A calycin/lipocalin lineage specific expansion was described in $R$. prolixus [14]. We found a significant enrichment in the three - Triatoma species, suggesting a gene family expansion that occurred in Triatomini after divergence from Rhodniini. Calycins form a large protein superfamily divided into lipocalins, fatty acid binding proteins, triabin, and thrombin inhibitors [53]. Of these, the lipocalin family (IPR002345) is the largest and functionally the most diverse. These are extracellular proteins sharing several recognition properties, such as ligand binding, receptor binding and the formation of complexes with other macromolecules, namely lipophilic compounds. Calycins constitute a preponderant component of several reduviid sialotranscriptomes, and are implicated in vasodilatation, anti-hemostasis and immunomodulation [15-18, 20, 31, 47, 51].

Lipocalin expansion was remarkable in T. infestans, where salivary lipocalins (clade IV), triafestin-like (clade V) and lipocalin/palidipin-like (clade VII) calycins groups doubled the numbers in T. pallidipennis and $T$. dimidiata (Additional file 2). Interestingly, we identified novel calycin groups, namely the intestinal and FABP clades (Fig. 3). Presumably, these novel calycins may be expressed in other organs or tissues than the salivary gland. A single FABP lipocalin, found in each analyzed Triatoma, is conserved in almost all metazoans and has been implicated in the day/night cycle modulation in D. melanogaster [54]. This opens the path for further structural, phylogenetic and functional characterization of the calycin/ lipocalin family. The rapid expansion of calycins in Triatoma, as well as their higher evolutionary rates (Fig. 5), is consistent with an adaptive role of this protein family in blood feeding and possibly other relevant biological processes beyond those in salivary gland.
An important difference among the three - Triatoma species was the higher number ( 5 to 8 -fold increase) of transcriptionally active transposable elements in $T$. infestans, particularly non-LTR retrotransposons (8 and 12fold increase) (Fig. 4a). This may be partially related to T. infestans genome size, which is one of the largest among all analyzed reduviids [55]; and genome size is associated with increased transposable and other repeated elements activity and content [56]. According to their putative taxonomic origin in RepBase database [35] and based on sequence identity, the majority of the nonLTR retrotransposons in $T$. infestans best-matched with bird CR1 non-LTR and showed higher sequence conservation than non-LTRs best-matching $R$. prolixus (Jockey, Nimb and Loa-type) (Fig. 4f). Horizontal transfer (HT) of DNA transposons from the blood meal source was recently proposed in $R$. prolixus [57]. Non-LTR HT is considered rare because it depends on unstable RNA intermediates. Nevertheless, evidence for non-LTR HT, including CR1 elements was documented in arthropods [58-60]. Also, endosymbiont to host genome gene transfer was demonstrated in the pea aphid A. pisum [61] and $R$. prolixus genomes [14]. Taken together, our results are consistent with recent HT events in $T$. infestans from birds (its major source of blood). Although we cannot rule out that non-LTR transcripts could derive from chicken blood used for feeding, these were not found in T. dimidiata and T. pallidipennis transcriptomes, which were fed from the same blood source under the same conditions. Further research and the availability of the $T$. infestans genome will contribute to clarify this issue.

Aiming at identifying signs of molecular adaptation, we first used protein sequence identity in Triatoma- $R$. prolixus 1:1 orthologs as an indicator of differences in evolutionary rates. Clustering of divergent proteins in a biological pathway, a function or a biological process is suggestive of high evolutionary rates of that particular process or function, and could guide the search for molecular adaptation. To document adaptation, a directed test for selection is required. In our work, an important limitation of using these approaches was that in some cases, the full ORF for each pair was unavailable. Nevertheless, the analysis of protein sequence conservation based on $\mathrm{Z}$ score comparisons and enrichment in the most divergent group suggest high evolutionary rates in oxidoreductase-mediated central metabolism, protease inhibitors, serine proteases in the three Triatomini, and in the calycin family at least in T. infestans, (Fig. 5, Additional file 7). In the case of serine proteases, this is a common finding in hematophagous insects [14, 30, 62], however high sequence divergence in immune response genes was also expected, but not detected. Lower evolutionary rates in core cellular processes such as protein biosynthesis, transport, signaling are consistent with 
other comparative genomics studies in insects in general and hematophagous insects in particular [30]. The finding that the protein conservation pattern in biological processes and cellular components do not correlate to phylogeny (Fig. 5b-c) may reflect that molecular function has a stronger correlation with structural ancestry (Fig. 5c), whereas biological process and cellular component depend on the convergence of multiple genes with different structural ancestry.

In D. melanogaster and other insects, gene adaptations in critical enzymes determining carbohydrate metabolic flux depend on their life history, latitudinal clines and flight capabilities [63, 64]. Although most of the glycolytic pathway enzymes were highly conserved in the three - Triatoma species, we identified sequence divergence in enzymes involved in anabolism, such as gluconeogenesis $(F b p)$, glycogen synthesis (Pgm1) and the PPP (Pgm2, Tald); and one enzyme that participates in glycolysis (Pyk) (Fig. 6, Additional file 8). Fbp generates fructose 6-phosphate a crucial regulator of metabolic flux towards the PPP. The opposite reaction is mediated by the 6-phosphofructokinase, which is critical for glycolysis required by flight muscles in insects. Additionally, the simultaneous activity of both enzymes is implicated in a "futile cycle", which generates heat from ATP hydrolysis, allowing the insect adaptation to temperate climates $[65,66]$. Molecular adaptation in $F b p$ and its relation to flight loss in Triatoma is a hypothesis worth of further investigation, especially in T. infestans, which is subjected to extreme day/night temperature variations.

Pgm1 is a critical enzyme catalyzing the interconversion of glucose 1-phosphate to glucose 6-phosphate, a critical step in the initiation of glycolysis and glycogen synthesis. Glycogen synthesis and storage is required to cope with different metabolic demands in insects. In Drosophila, adaptive mutations in the Pgm1 locus show latitudinal clinal variation correlating with increased glycogen storage in temperate regions [67, 68]. Glycogen storage increases after blood meal in $R$. prolixus, is required for oogenesis and is completely depleted after prolonged starvation periods [69-72] (Fig. 6). Based on these, our observations could represent molecular adaptation in the Pgm1 locus in reduviids, associated with an optimization in glycogen metabolic flux to cope with prolonged starvation and to achieve reproductive success.

The PPP, an early branch from glycolysis, is a major source of NADPH (oxidative phase) required for fatty acid synthesis and reactive oxygen species (ROS) scavenging via reduced glutathione. In its non-oxidative phase, it generates precursors for the synthesis of ribonucleotides. In $D$. melanogaster, an enhanced activity of the
PPP oxidative phase induced by glucose 6-phosphate dehydrogenase (G6pd) overexpression, increases the levels of NADPH and extends the fly lifespan [73]. Among the Triatoma PPP enzymes, Pgm2 and Tald belonged to the most divergent group, both mediate reactions in the non-oxidative phase. Ribose-5-phosphate isomerase (Rpi) generates the substrate for Pgm2, which in turn promotes ribonucleotide synthesis (Fig. 6). Rpi inhibition extends Drosophila life span [74]. Tald deficiency induces a starvation-like, mitochondrial stress, autophagy and extended life span in Caenorhabditis elegans [75]. This opens the possibility that the non-oxidative PPP may be implicated in contributing to the extended life span of reduviids.

The only glycolytic enzyme found in the most divergent group, $P y k$ mediates the last step of glycolysis, catalyzing the bidirectional inter-conversion between phosphoenolpyruvate and pyruvate (Fig. 6). Fructose 1, 6-bisphosphate is an allosteric activator of $P y k$. Thus, gluconeogenesis and high $F b p$ activity suppresses $P y k$ [76]. The possibility that genetic variations in $F b p$ and $P y k$ may be functionally related deserves further study.

A clear overrepresentation of components of the respiratory chain complexes in the most divergent group was identified (Additional file 7); however, no evidence for positive selection was found using codon-based Ztests, which are highly specific, although less sensitive for detecting selection. We reasoned that OxPhos proteins may be evolving under complex mechanisms of selection, and that signals for positive selection could be masked by an overwhelming number of sites under negative selection. Thus, we performed the positionbased selection test with HyPhy. Although we did not find statistical significance in a per codon basis, a statistically significant bias towards positive $\mathrm{dN}-\mathrm{dS}$ values was documented in the mitochondrial genome-encoded core subunits, and the $\beta$ subcomplex subunits of NADH dehydrogenase complex (I); Cox7A subunit of the cytochrome oxidase C complex (IV) and the $F_{O}$ - ATP synthase complex (V) subunits (Fig. 7), indicating increased likelihood of positive selection. Statistical significance in this method is highly dependent on the number of sequences included in the comparison (only four in this case), which could explain the lack of statistical significance in codons with positive $\mathrm{dN}-\mathrm{dS}$ ).

The NDUFA11 ( $\alpha$ subcomplex, NADH dehydrogenase), NDUFB4, NDUFB8, NDUFB9 ( $\beta$ subcomplex of NADH dehydrogenase) and Cox7A2 mammalian homologs are implicated in the formation of respiratory chain "supercomplexes", which may be required for adequate mitochondrial function [77]. Moreover, reactive oxygen species participate in ageing process in many metazoan models. The production of ROS by reverse electron transport, mediated by complex I or RNAi-mediated 
inhibition of complex I, III, IV and V, is associated with extended life span in Drosophila [78, 79]. Also, several complex I, IV and V mitochondria-encoded genes evolve under positive selection in flying insects, including hemipterans, but not in flightless insect orders [80].

We have based our interpretation of position-based selection test $p$ values in the context of biological plausibility (i.e., examples of selection in OxPhos genes in other insects), a coherent effect in protein divergence in certain functionally related genes, and an equally coherent biased distribution of $\mathrm{dN}-\mathrm{dS}$ of certain, but not all OxPhos sub-complexes (i.e., Fo but not F1 ATP synthase) to suggest, although not prove, the likelihood of selection in complexes I, IV and V. These observations suggest that triatomines underwent molecular adaptations in oxidative phosphorylation which may have resulted in optimization of energy metabolism and ROS generation balance. This optimization may allow these insects, on the one hand, to cope with prolonged starvation periods and on the other, the production the high amounts of energy demanded during hematophagy [81]. Their enhanced capacity to scavenge ROS by NADPH could contribute to their extended lifespan and might also be linked to their vectorial capacity, as redox status has been suggested to trigger changes in the life cycle of Trypanosoma cruzi in the vector [82].

\section{Conclusions}

Overall, our results documented a set of shared gene expansions in triatomines possibly related to biological adaptations to their lifestyles and diverse ecological niches. Protease inhibitor and calycin-coding gene expansions points at rapidly evolving processes of protease regulation and hematophagy, which require further research for a better understanding of their role in vectorial capacity. Higher evolutionary rates in critical enzymes that exert metabolic flux control towards glycogen synthesis and the PPP, in oxidative phosphorylation complexes, and the expansion of the thioredoxin fold-containing proteins could represent genetic adaptations favoring hallmarks of triatomine life styles. Particularly, glycogen storage to cope with prolonged starvation and reproductive success, antioxidant mechanisms to cope with the oxidative stress favoring longevity, and optimization of aerobic metabolism to meet the energetic demands of hematophagy. Overall, this work represents a source of novel hypotheses related to triatomine biology to guide experimental testing.

\section{Methods}

\section{Insect rearing}

Colonies of $T$. dimidiata (colony 0252 from Tegucigalpa, Honduras), T. infestans (colony X32 from Santiago del Estero, Argentina) and T. pallidipennis (colony 0230 from Mexico) established in Centro Nacional de Chagas, Córdoba, Argentina were reared in the Centro Regional de Estudios Genómicos (CREG), Universidad Nacional de La Plata (UNLP) and the Centro de Bioinvestigaciones, Universidad Nacional del Noroeste de Buenos Aires (UNNOBA), at $28{ }^{\circ} \mathrm{C}$ and a partial humidity of $70 \%$ with a $12 \mathrm{~h}$ light/dark schedule. Insects were regularly fed using an artificial feeder and chicken blood. Insect handling was performed in accordance to the World Health Organization protocol [5].

\section{Transcriptome preparation and sequencing}

A mixture of RNA derived from different life-cycle stages of $T$. infestans, $T$. dimidiata and $T$. pallidipennis was used to generate a normalized cDNA library for each species as described [83]. Libraries were barcoded and subjected to the shotgun sequencing protocol using the GS FLX+ (454-Roche). Raw sequence datasets are available at the NCBI-SRA: T. infestans (SRX2600754), T. dimidiata (SRX2600753) and T. pallidipennis (SRX2600752).

\section{Data filtering, trimming and assembly}

Raw reads were analyzed with PRINSEQ [84] and filtered according to length, sequence complexity and quality. Each library was subjected to de novo assembly with the GS DeNovo assembler v.2.8 software in cDNA mode using the default parameters, and including the adaptor sequences for trimming. The assembled sequences dataset are available at the NCBI-TSA (GFMK00000000, GFMC00000000, GFMJ01000000). The non-assembled reads were mapped to the $R$. prolixus genome (Rhodnius-prolixus-CDC_SCAFFOLDS_ RproC3.fa) using BLASTN and proteome (Rhodniusprolixus-CDC_PEPTIDES_RproC3.2.fa) using BLASTX. Non-redundant mapped reads to either database were included as singletons in to the assembled dataset (full dataset).

To mitigate the potential effects of redundancy in the full_dataset in the assessment of statistical representation of GO and InterPro annotations, two additional sequence databases for each species were built. A non-redundant database ( $n r_{-}$dataset) discards alternative isotigs belonging to the same isogroup or unigene, by keeping the largest isotig (transcript) per isogroup. The third database includes only nonredundant isotigs (isotig_dataset). An additional transcriptome database was created for each Triatoma species, by mapping raw reads to the $\mathrm{R}$. prolixus predicted transcript dataset (Rhodnius-prolixus-CDC_ TRANSCRIPTS_RproC3.2.fa) with the GS (Newbler) Mapper v.2.8. This dataset (RproC3.2_mapped) was used only for the identification of metabolic pathways enzyme-coding transcripts (Fig. 6. Additional file 8). 
To estimate the proportion of reads coded by the mitochondrial genome and to identify mitochondrialencoded genes, the GS Mapper v 2.8 was used to map raw reads of each species to the $T$. dimidiata mitochondrial genome (Genbank accession: $\mathrm{NC}_{-}$ 002609.1) [22]. The same was done for the Triatoma virus genome (Genbank accession: NC_003783.1) (Table 2). All datasets used in this work are available in http://201.131.57.23:8080/data/triatoma.

\section{Transcriptome completeness analysis}

The assembled dataset for each species was used to identify the proportion of the core eukaryotic genome coverage, as described [83]. We used HMM profiles for 458 core eukaryotic proteins as provided by the CEGMA dataset [23] and HMMER3 searches with the hmmscan command and the -T 40 and -domT 40 filters, as described in [62]. Following the same approach, a Benchmarking Universal Single-Copy Orthologs (BUSCO) sets for arthropod [24] was used to assess transcriptome dataset completeness.

To estimate the proportion of each transcriptome database that is homologous to the $R$. prolixus predicted proteome [14], we used NCBI-BLASTX (-e 1. OE-05). Putative 1:1 orthologue identification between $R$. prolixus and the three - Triatoma transcriptomes was done using the BLAST best reciprocal hit strategy as described [62].

\section{Insect proteome comparisons}

The full_dataset transcriptome assemblies were used to find homolog proteins using BLASTX (cut-off -e 1.0E05) in the following arthropod proteome databases (Ixodes scapularis, Pediculus humanus, Lutzomya longipalpis, R. prolixus, Phlebotomus papatasi, Culex quinquefaciatus, Anopheles gambiae, Aedes aegypti, and Glossina morsitans) downloaded from VectorBase [85], as well as D. melanogaster [86] and Ac. pisum [61].

\section{Gene ontology and Interpro annotation}

To classify transcripts according to their putative biological process, molecular function and structural relationships (protein conserved domains), these were analyzed according to Gene Ontology (GO) "terms" [87] and InterPro [25] annotations using the software package BLAST2Go Pro [26]. For the initial step, BLASTX against the NCBI-nr database, with a cut-off $e$ value of 1.0E-6, was used. Once annotated, Gene Ontology term enrichment using as input the isotig_dataset was performed by means of Fisher's exact test followed by a $P$ value adjustment to correct for multiple testing with the Benjamini-Hochberg method (cut-off FDR $<0.05$ ).

\section{Identification of putative gene family expansions}

The absolute count of InterPro annotations in the non-redundant isotig_dataset was used to estimate enrichment, using the $R$. prolixus InterPro annotations as reference. To avoid spurious enrichments, repeated InterPro ID's in the same transcripts/gene were counted only once. A Fisher Exact Test, a 2 X 2 contingency table was built between the number of annotated and the non-annotated transcripts for a given IPR entry for each Triatoma species (test), as well as for $R$. prolixus (reference). The $\mathrm{H}_{0}$ is that there is no difference in the probability distribution between species and belonging to a given IPR entry. Given that transcript underrepresentation in transcriptome analysis in meaningless due the lack of certainty of completeness, emphasis was put in enrichment of IPR terms in Triatoma $(\mathrm{Ha})$ by using a one-sided Fisher's test with the $\mathrm{R}$ function fisher.test (c, alternative = "greater"), which indicates an enrichment in the test dataset. The function fisher.test $(\mathrm{c}$, alternative $=$ "less") was also used to complement the enrichment analysis within Triatoma species, which indicates depletion in the reference dataset. $\mathrm{P}$ value adjustment with the Benjamini-Hochberg method was performed to correct for multiple testing using the $\mathrm{R}$ function $\mathrm{p}$. $\operatorname{adjust}(\mathrm{p}, \operatorname{method}=$ "bh", $\mathrm{n}=$ length(p)). A False Discovery Rate $(\mathrm{FDR})<0.05$ was considered as a significant enrichment [88]. Individual protein family analysis was complemented with local InterProScan analysis [25] or HMMER3 searches using specific PFAM profiles [89].

\section{Transposable element analysis}

Analysis of expressed transposable elements (TEs) was performed with local NCBI-BLASTN, with a cut-off $e$ value of $1.0 \mathrm{E}-6$ against the RepBase database, Version 21.08 [35]. Matches with alignment lengths lesser than $100 \mathrm{bps}$ were discarded. Analysis was only focused in DNA transposons (Class II), LTR and non-LTR retrotransposons.

\section{Cluster analysis of the calycin/lipocalin family}

To analyze the global phylogenetic relationships of our calycin/lipocalin ortholog predictions, we performed a clustering analysis with CLANS software [32]. CLANS is a useful tool to generate 3D clustering many sequences based on their sequence similarity. It uses a variant of the Fruchterman and Reingold graph layout algorithm to generate graphs providing graphical representation of pairwise sequence similarities. Sequences are represented by vertices in the graph, BLAST/PSIBLAST high scoring segment pairs (HSPs) are shown as edges connecting vertices and provide attractive forces proportional to the negative algorithm of the HSP's P -value. In 
this way, similar sequences reproducibly cluster after a few iterations. The parameters used for the analysis were as follows: PSI-BLAST as sequence-similarity-based approach, BLOSUM62 matrix, P-value cutoff of $1 \mathrm{e}-37$, repuls $=2$ and attract $=2$.

\section{Estimation of divergence in ortholog pairs according to $G O$} The sequence identity (\%) for each 1:1 $R$. prolixus Triatoma ortholog pair identified by BLAST - best reciprocal hit strategy (isotig_dataset) was grouped according to Gene Ontology biological process, molecular function and cellular component categories (Level 3). Median \% identity for each category was used to calculate $\mathrm{Z}$ values (number of standard deviations from the \% identity mean) by subtracting the overall median \% identity (all GO annotated orthologs). Statistical significance $(p<0.05)$ was determined with Kruskal-Wallis one-way ANOVA test with Dunn's correction for multiple comparisons. $\mathrm{Z}$ values were hierarchically clustered using Cluster 3.0 [90] according to species and graphically represented as heatmaps using Java TreeView 1.1.6r4 (http://jtreeview. sourceforge.net).

To study protein sequence divergence according to more specific GO terms, the whole set of GO 1:1 orthologs (isotig-dataset) was subdivided into equal terciles according to \% identity (BLASTx) (i.e. more, average and less-conserved terciles or groups) (Additional file 6). To identify more specific GO categories that were enriched in the less conserved group, a Fisher Exact Test was performed using the GO annotations in the most conserved group as reference using BLAST2Go Pro [26]. $P$ values were adjusted for multiple testing using the BenjaminiHochberg method. A FDR $<0.05$ was considered significant.

\section{Selection analysis of the respiratory chain complexes}

To test selection in coding genes of the oxidative phosphorylation complexes, orthologs nucleotide sequences of the four species were codon-aligned. All ambiguous positions and gaps were removed for each sequence pair. Two approaches were used. The first was a codon-based Z-test of positive selection in which the variance of the difference of the numbers of synonymous (dS) and nonsynonymous $(\mathrm{dN})$ substitutions per site was computed using the Nei-Gojobori method [91]. In the second approach, nucleotide sequences were subjected to a codonby-codon Maximum Likelihood analysis with the HyPhy package [42] using the Muse-Gaut model [92] of codon substitution and Felsenstein model [93] of nucleotide substitution. The median of normalized $\mathrm{dN}-\mathrm{dS}$ for each variant position was calculated and plotted according to increasing median value. Statistical significance was evaluated with a Kruskal-Wallis test with Dunn's multiple comparisons correction between the sequence with the lowest-median $\mathrm{dN}-\mathrm{dS}$ and the remaining sequences. Evolutionary analyses were conducted in MEGA7 [94].

\section{Additional files}

Additional file 1: InterProScan analysis of Pacifastin domain containing proteins in Reduviids (IPR008037). Data for Fig. 2. (XLS 40 kb)

Additional file 2: Analysis of the calycin family in Triatoma. Data for Fig. 3. (XLS $58 \mathrm{~kb})$

Additional file 3: Transcriptionally active transposable elements in Triatoma. BLASTn analysis search in the RepBase database. Data for Fig. 4. (XLS 64 kb)

Additional file 4: Transcripts coding for odorant receptors and odorant binding proteins in Triatoma. (XLS $78 \mathrm{~kb}$ )

Additional file 5: Protein sequence divergence in putative 1:1 orthologs according to Gene Ontology terms (Level 3). Isotig dataset. Data for Fig. 5. (XLS 54 kb)

Additional file 6: Sequence identity distribution for $R$. prolixus-Triatoma 1:1 putative orthologs divided into equal terciles or groups. (PDF $450 \mathrm{~kb}$ )

Additional file 7: Gene ontology term enrichment in divergent vs. conserved group putative 1:1 orthologs (isotig dataset). (XLS 43 kb)

Additional file 8: Identification of Glycolysis, gluconeogenesis and pentose-phosphate putative orthologs and classification according to protein sequence conservation (RproC3.2_mapped dataset. Data for Fig. 6. (XLS 62 kb)

Additional file 9: Oxidative phosphorylation. Identification of respiratory complex subunit putative orthologs and classification according to protein sequence conservation ( $n r$-dataset and mitochondrial_mapped datasets. Data for Fig. 7) (XLS 69 kb)

\section{Abbreviations}

ERV: Endogenous retrovirus; FABP: Fatty acid binding protein; FDR: False discovery rate; HT: Horizontal transfer; LTR: Long terminal repeat; nonLTR: Non-long terminal repeat; OBP: Odorant binding protein; OxPhos: Oxidative phosphorylation; PPP: Pentose phosphate pathway; ROS: Reactive oxygen species; T. cruzi: Trypanosoma cruzi; TCA: Tricarboxylic acid; TE: Transposable element; WWFC: von Willebrand factor, type C domain; WWFD: von Willebrand factor, type D domain

\section{Acknowledgements}

We are thankful to Raúl Stariolo and Patricia Lobbia from Centro de Referencia de Vectores (CeReVe), Argentina, for their generous help and providing insects; Andrés Aguilar (LANGEBIO) for support in sequencing data QC, Gilberto Sánchez (CISEI-INSP) for statistical support and José Manuel Latorre-Estivalis (CREG-UNLP) for support in odorant receptor and OBP analysis. We also thank the anonymous reviewers for their thorough revision, their comments and suggestions.

\section{Funding}

AL and RRP are Investigators of Consejo Nacional de Ciencia y Tecnología (CONICET). This work was supported by Agencia Nacional de Promoción de Ciencia y Técnica (PICT-2013-1554), International Cooperation Grant from CONICET (\# 999/2013) and Ministerio de Relaciones Exteriores de Argentina (FO-AR 5790) to RRP; Concejo Nacional de Ciencia y Tecnología (CONACyT) \# 19612 and Agencia Mexicana de Cooperación Internacional para el Desarrollo (AMEXCID) to MHR. Scholarship awards to MB, CONICET; VP, Agencia Nacional de Promoción de Ciencia y Técnica; and FAZE, CONACyT.

\section{Availability of data and materials}

Raw sequencing data and de novo assembled transcriptomes are available at NCBI-SRA (SRX2600754, SRX2600753 and SRX2600752) and NCBI-TSA (GFMK00000000, GFMC00000000, GFMJ01000000). All sequence datasets and annotations used in this manuscript are available at http://

201.131.57.23:8080/data/triatoma. 


\section{Authors' contributions}

JMB conceived the study, designed data analysis approach, analyzed data and wrote the manuscript. AL generated sequencing libraries, analyzed data and wrote the manuscript. JTS generated sequencing libraries and sequenced them. MB, VP, FAZE and EEGL analyzed data. MHR and RRP conceived the study, analyzed the results and wrote the manuscript. All authors read and approved the manuscript for publication.

\section{Ethics approval and consent to participate}

The present work did not involve research with human subjects. Insect handling was performed in accordance to the World Health Organization protocol [5] and INSP and CONICET guidelines regarding animal care. This work was approved by UNOBA, UNLP and INSP research committees.

\section{Competing interests}

The authors declare that they have no competing interests.

\section{Publisher's Note}

Springer Nature remains neutral with regard to jurisdictional claims in published maps and institutional affiliations.

\section{Author details}

${ }^{1}$ Centro de Investigación Sobre Enfermedades Infecciosas, Instituto Nacional de Salud Pública, Cuernavaca, México. ${ }^{2}$ Centro de Bioinvestigaciones (CeBio) and Centro de Investigación y Transferencia del Noroeste de Buenos Aires (CITNOBA-CONICET), Universidad Nacional del Noroeste de la Provincia de Buenos Aires, Pergamino, Argentina. ${ }^{3}$ Laboratorio de Genética y Genómica Funcional. Centro Regional de Estudios Genómicos. Facultad de Ciencias Exactas, Universidad Nacional de La Plata, La Plata, Argentina.

Received: 24 June 2017 Accepted: 18 April 2018

Published online: 27 April 2018

\section{References}

1. Lent H WP: Revision of the Triatominae (Hemiptera, Reduviidae), and their significance as vectors of Chagas' disease, vol 163; 1979.

2. Justi SA, Galvao C. The evolutionary origin of diversity in Chagas disease vectors. Trends Parasitol. 2017;33(1):42-52.

3. Hwang WS, Weirauch C. Evolutionary history of assassin bugs (insecta: hemiptera: Reduviidae): insights from divergence dating and ancestral state reconstruction. PLoS One. 2012;7(9):e45523.

4. Gaunt M, Miles M. The Ecotopes and evolution of Triatomine bugs (Triatominae) and their associated trypanosomes. Mem Inst Oswaldo Cruz. 2000;95(4):557.

5. WHO. Control of Chagas disease. World Health Organization Tech Rep Ser. 2002;905:i-vi. 1-109, back cover

6. Fitzpatrick S, Feliciangeli MD, Sanchez-Martin MJ, Monteiro FA, Miles MA. Molecular genetics reveal that silvatic Rhodnius prolixus do colonise rural houses. PLoS Negl Trop Dis. 2008;2(4):e210.

7. Rassi A Jr, Rassi A, Marin-Neto JA. Chagas disease. Lancet. 2010;375(9723): $1388-402$.

8. Zeledon R, Guardia VM, Zuniga A, Swartzwelder JC. Biology and ethology of Triatoma dimidiata (Latreille, 1811). II. Life span of adults and fecundity and fertility of females. J Med Entomol. 1970;7(4):462-9.

9. Zeledon R, Guardia VM, Zuniga A, Swartzwelder JC. Biology and ethology of Triatoma dimidiata (Latreille, 1811). I. Life cycle, amount of blood ingested, resistance of starvation, and size of adults. J Med Entomol. 1970;7(3):313-9.

10. Monroy MC, Bustamante DM, Rodas AG, Enriquez ME, Rosales RG. Habitats, dispersion and invasion of sylvatic Triatoma dimidiata (Hemiptera: Reduviidae: Triatominae) in Peten, Guatemala. J Med Entomol. 2003;40(6): 800-6.

11. Ramsey JM, Peterson AT, Carmona-Castro O, Moo-Llanes DA, Nakazawa Y, Butrick M, Tun-Ku E, la Cruz-Felix K, Ibarra-Cerdena CN. Atlas of Mexican Triatominae (Reduviidae: Hemiptera) and vector transmission of Chagas disease. Mem Inst Oswaldo Cruz. 2015;110(3):339-52.

12. Ramsey JM, Ordonez R, Cruz-Celis A, Alvear AL, Chavez V, Lopez R, Pintor JR, Gama F, Carrillo S. Distribution of domestic triatominae and stratification of Chagas disease transmission in Oaxaca, Mexico. Med Vet Entomol. 2000; 14(1):19-30
13. Gurtler RE. Sustainability of vector control strategies in the gran Chaco region: current challenges and possible approaches. Mem Inst Oswaldo Cruz. 2009;104(Suppl 1):52-9.

14. Mesquita RD, Vionette-Amaral RJ, Lowenberger C, Rivera-Pomar R, Monteiro FA, Minx P, Spieth J, Carvalho AB, Panzera F, Lawson D, et al. Genome of Rhodnius prolixus, an insect vector of Chagas disease, reveals unique adaptations to hematophagy and parasite infection. Proc Natl Acad Sci U S A. 2015;112(48):14936-41.

15. Ribeiro JMC, Assumpcao TCF, Pham VM, Francischetti IMB, Reisenman CE. An insight into the Sialotranscriptome of Triatoma rubida (Hemiptera: Heteroptera). J Med Entomol. 2012;49(3):563-72.

16. Assumpcao TC, Francischetti IM, Andersen JF, Schwarz A, Santana JM, Ribeiro JM. An insight into the sialome of the blood-sucking bug Triatoma infestans, a vector of Chagas' disease. Insect Biochem Mol Biol. 2008;38(2):213-32.

17. Assumpcao TC, Eaton DP, Pham VM, Francischetti IM, Aoki V, Hans-Filho G, Rivitti EA, Valenzuela JG, Diaz LA, Ribeiro JM. An insight into the sialotranscriptome of Triatoma matogrossensis, a kissing bug associated with fogo selvagem in South America. American J Trop Med Hygiene. 2012; 86(6):1005-14

18. Ribeiro JM, Schwarz A, Francischetti IM. A deep insight into the Sialotranscriptome of the Chagas disease vector, Panstrongylus megistus (Hemiptera: Heteroptera). J Med Entomol. 2015;52(3):351-8.

19. Traverso L, Lavore A, Sierra I, Palacio V, Martinez-Barnetche J, Latorre-Estivalis JM, Mougabure-Cueto G, Francini F, Lorenzo MG, Rodriguez MH, et al. Comparative and functional triatomine genomics reveals reductions and expansions in insecticide resistance-related gene families. PLoS Negl Trop Dis. 2017;11(2):e0005313.

20. Hernandez-Vargas MJ, Santibanez-Lopez CE, Corzo G. An insight into the Triabin protein family of American hematophagous Reduviids: functional, structural and phylogenetic analysis. Toxins. 2016;8(2):44

21. Marchant A, Mougel F, Almeida C, Jacquin-Joly E, Costa J, Harry M. De novo transcriptome assembly for a non-model species, the bloodsucking bug Triatoma brasiliensis, a vector of Chagas disease. Genetica. 2015:143(2):225-39.

22. Dotson EM, Beard CB. Sequence and organization of the mitochondrial genome of the Chagas disease vector, Triatoma dimidiata. Insect Mol Biol. 2001;10(3):205-15.

23. Parra G, Bradnam K, Korf I. CEGMA: a pipeline to accurately annotate core genes in eukaryotic genomes. Bioinformatics. 2007;23(9):1061-7.

24. Simao FA, Waterhouse RM, loannidis P, Kriventseva EV, Zdobnov EM. BUSCO: assessing genome assembly and annotation completeness with single-copy orthologs. Bioinformatics. 2015;31(19):3210-2.

25. Jones $P$, Binns D, Chang HY, Fraser M, Li W, McAnulla C, McWilliam H, Maslen J, Mitchell A, Nuka G, et al. InterProScan 5: genome-scale protein function classification. Bioinformatics. 2014;30(9):1236-40.

26. Gotz S, Garcia-Gomez JM, Terol J, Williams TD, Nagaraj SH, Nueda MJ, Robles M, Talon M, Dopazo J, Conesa A. High-throughput functional annotation and data mining with the Blast2GO suite. Nucleic Acids Res. 2008:36(10):3420-35.

27. Breugelmans B, Simonet $G$, van Hoef V, Van Soest S, Vanden Broeck J. Pacifastin-related peptides: structural and functional characteristics of a family of serine peptidase inhibitors. Peptides. 2009;30(3):622-32.

28. Wallin $H$, Bjarnadottir $M$, Vogel LK, Wasselius J, Ekstrom U Abrahamson M. Cystatins-extra- and intracellular cysteine protease inhibitors: high-level secretion and uptake of cystatin $C$ in human neuroblastoma cells. Biochimie. 2010;92(11):1625-34.

29. Turk V, Stoka V, Turk D. Cystatins: biochemical and structural properties, and medical relevance. Front Biosci. 2008;13:5406-20.

30. Neafsey DE, Waterhouse RM, Abai MR, Aganezov SS, Alekseyev MA, Allen JE, Amon J, Arca B, Arensburger P, Artemov G, et al. Mosquito genomics. Highly evolvable malaria vectors: the genomes of 16 Anopheles mosquitoes. Science. 2015:347(6217):1258522.

31. Kato H, Jochim RC, Gomez EA, Sakoda R, Iwata H, Valenzuela JG, Hashiguchi $Y$. A repertoire of the dominant transcripts from the salivary glands of the blood-sucking bug, Triatoma dimidiata, a vector of Chagas disease. Infect Genet Evol. 2010;10(2):184-91.

32. Frickey $\mathrm{T}$, Lupas A. CLANS: a java application for visualizing protein families based on pairwise similarity. Bioinformatics. 2004;20(18):3702-4.

33. Montfort WR, Weichsel A, Andersen JF. Nitrophorins and related antihemostatic lipocalins from Rhodnius prolixus and other bloodsucking arthropods. Biochim Biophys Acta. 2000;1482(1-2):110-8. 
34. Ribeiro JM, Genta FA, Sorgine MH, Logullo R, Mesquita RD, Paiva-Silva GO, Majerowicz D, Medeiros M, Koerich L, Terra WR, et al. An insight into the transcriptome of the digestive tract of the bloodsucking bug, Rhodnius prolixus. PLoS Negl Trop Dis. 2014;8(1):e2594.

35. Bao W, Kojima KK, Kohany O. Repbase update, a database of repetitive elements in eukaryotic genomes. Mob DNA. 2015;6:11.

36. Potter CJ. Stop the biting: targeting a mosquito's sense of smell. Cell. 2014; 156(5):878-81.

37. Rebers JE, Willis $\mathrm{JH}$. A conserved domain in arthropod cuticular proteins binds chitin. Insect Biochem Mol Biol. 2001;31(11):1083-93.

38. Suetake T, Tsuda S, Kawabata S, Miura K, Iwanaga S, Hikichi K, Nitta K, Kawano K. Chitin-binding proteins in invertebrates and plants comprise a common chitin-binding structural motif. J Biol Chem. 2000;275(24):17929-32.

39. Alvarenga ES, Mansur JF, Justi SA, Figueira-Mansur J, Dos Santos VM, Lopez SG, Masuda H, Lara FA, Melo AC, Moreira MF. Chitin is a component of the Rhodnius prolixus midgut. Insect Biochem Mol Biol. 2016;69:61-70.

40. Soares JB, Gaviraghi A, Oliveira MF. Mitochondrial physiology in the major arbovirus vector Aedes aegypti: substrate preferences and sexual differences define respiratory capacity and superoxide production. PLoS One. 2015; 10(3):e0120600.

41. Brand MD. Mitochondrial generation of superoxide and hydrogen peroxide as the source of mitochondrial redox signaling. Free Radic Biol Med. 2016; 100:14-31.

42. Kosakovsky Pond SL, Frost SD. Not so different after all: a comparison of methods for detecting amino acid sites under selection. Mol Biol Evol. 2005; 22(5):1208-22.

43. Waterhouse RM. A maturing understanding of the composition of the insect gene repertoire. Current Opin Insect Sci. 2015;7:15-23.

44. Feyereisen R. Arthropod CYPomes illustrate the tempo and mode in P450 evolution. Biochim Biophys Acta. 2011;1814(1):19-28.

45. Liang Z, Sottrup-Jensen L, Aspan A, Hall M, Soderhall K. Pacifastin, a novel 155-kDa heterodimeric proteinase inhibitor containing a unique transferrin chain. Proc Natl Acad Sci U S A. 1997;94(13):6682-7.

46. Breugelmans B, Simonet G, van Hoef V, Van Soest S, Broeck JV. Identification, distribution and molecular evolution of the pacifastin gene family in Metazoa. BMC Evol Biol. 2009;9:97.

47. Schwarz A, Medrano-Mercado N, Schaub GA, Struchiner CJ, Bargues MD, Levy MZ, Ribeiro JM. An updated insight into the Sialotranscriptome of Triatoma infestans: developmental stage and geographic variations. PLoS Negl Trop Dis. 2014;8(12):e3372.

48. de Marco R, Lovato DV, Torquato RJ, Clara RO, Buarque DS, Tanaka AS. The first pacifastin elastase inhibitor characterized from a blood sucking animal. Peptides. 2010;31(7):1280-6.

49. Kotsyfakis M, Karim S, Andersen JF, Mather TN, Ribeiro JM. Selective cysteine protease inhibition contributes to blood-feeding success of the tick Ixodes scapularis. J Biol Chem. 2007;282(40):29256-63.

50. Kotsyfakis M, Sa-Nunes A, Francischetti IM, Mather TN, Andersen JF, Ribeiro JM. Antiinflammatory and immunosuppressive activity of sialostatin L, a salivary cystatin from the tick Ixodes scapularis. J Biol Chem. 2006;281(36):26298-307.

51. Assumpcao TC, Charneau S, Santiago PB, Francischetti IM, Meng Z, Araujo CN, Pham VM, Queiroz RM, de Castro CN, Ricart CA, et al. Insight into the salivary transcriptome and proteome of Dipetalogaster maxima. J Proteome Res. 2011;10(2):669-79.

52. Buarque DS, Spindola LM, Martins RM, Braz GR, Tanaka AS. Tigutcystatin, a cysteine protease inhibitor from Triatoma infestans midgut expressed in response to Trypanosoma cruzi. Biochem Biophys Res Commun. 2011:413(2):241-7.

53. Flower DR, North AC, Sansom CE. The lipocalin protein family: structural and sequence overview. Biochim Biophys Acta. 2000;1482(1-2):9-24.

54. Gerstner JR, Vanderheyden WM, Shaw PJ, Landry CF, Yin JC. Fatty-acid binding proteins modulate sleep and enhance long-term memory consolidation in Drosophila. PLoS One. 2011;6(1):e15890.

55. Panzera F, Ferrandis I, Ramsey J, Salazar-Schettino PM, Cabrera M, Monroy C, Bargues MD, Mas-Coma S, O'Connor JE, Angulo VM, et al. Genome size determination in chagas disease transmitting bugs (hemiptera-triatominae) by flow cytometry. Am J Tropical Med Hyg. 2007;76(3):516-21.

56. Habibi L, Pedram M, AmirPhirozy A, Bonyadi K. Mobile DNA elements: the seeds of organic complexity on earth. DNA Cell Biol. 2015;34(10): 597-609.
57. Gilbert C, Schaack S, Pace JK 2nd, Brindley PJ, Feschotte C. A role for hostparasite interactions in the horizontal transfer of transposons across phyla. Nature. 2010;464(7293):1347-50.

58. Sormacheva I, Smyshlyaev G, Mayorov V, Blinov A, Novikov A, Novikova O. Vertical evolution and horizontal transfer of CR1 non-LTR retrotransposons and Tc1/mariner DNA transposons in Lepidoptera species. Mol Biol Evol. 2012;29(12):3685-702.

59. Novikova O, Sliwinska E, Fet V, Settele J, Blinov A, Woyciechowski M. CR1 clade of non-LTR retrotransposons from Maculinea butterflies (Lepidoptera: Lycaenidae): evidence for recent horizontal transmission. BMC Evol Biol. 2007:7:93

60. Biedler JK, Chen X, Tu Z. Horizontal transmission of an R4 clade non-long terminal repeat retrotransposon between the divergent Aedes and Anopheles mosquito genera. Insect Mol Biol. 2015;24(3):331-7.

61. International Aphid Genomics C. Genome sequence of the pea aphid Acyrthosiphon pisum. PLoS Biol. 2010;8(2):e1000313.

62. Martinez-Barnetche J, Gomez-Barreto RE, Ovilla-Munoz M, Tellez-Sosa J, Garcia Lopez DE, Dinglasan RR, Ubaida Mohien C, MacCallum RM, Redmond SN, Gibbons JG, et al. Transcriptome of the adult female malaria mosquito vector Anopheles albimanus. BMC Genomics. 2012;13:207.

63. Eanes WF. Molecular population genetics and selection in the glycolytic pathway. J Exp Biol. 2011;214(Pt 2):165-71.

64. Zera AJ. Microevolution of intermediary metabolism: evolutionary genetics meets metabolic biochemistry. J Exp Biol. 2011;214(Pt 2):179-90.

65. Leite A, Neto JA, Leyton JF, Crivellaro O, el-Dorry HA. Phosphofructokinase from bumblebee flight muscle. Molecular and catalytic properties and role of the enzyme in regulation of the fructose 6-phosphate/fructose 1,6bisphosphate cycle. J Biol Chem. 1988;263(33):17527-33.

66. Staples JF, Koen EL, Laverty TM. Futile cycle' enzymes in the flight muscles of north American bumblebees. J Exp Biol. 2004;207(Pt 5):749-54.

67. Verrelli BC, Eanes WF. The functional impact of Pgm amino acid polymorphism on glycogen content in Drosophila melanogaster. Genetics. 2001;159(1):201-10.

68. Verrelli BC, Eanes WF. Clinal variation for amino acid polymorphisms at the Pgm locus in Drosophila melanogaster. Genetics. 2001;157(4):1649-63.

69. Wigglesworth VB. Cytological changes in the fat body of Rhodnius during starvation, feeding and oxygen want. J Cell Sci. 1967;2(2):243-56.

70. Santos R, Mariano AC, Rosas-Oliveira R, Pascarelli B, Machado EA, MeyerFernandes JR, Gondim KC. Carbohydrate accumulation and utilization by oocytes of Rhodnius prolixus. Arch Insect Biochem Physiol. 2008;67(2):55-62.

71. Mariano AC, Santos R, Gonzalez MS, Feder D, Machado EA, Pascarelli B, Gondim KC, Meyer-Fernandes JR. Synthesis and mobilization of glycogen and trehalose in adult male Rhodnius prolixus. Arch Insect Biochem Physiol. 2009;72(1):1-15.

72. Mury FB, Lugon MD, RN DAF, Silva JR, Berni M, Araujo HM, Fontenele MR, Abreu LA, Dansa M, Braz G, et al. Glycogen synthase Kinase-3 is involved in glycogen metabolism control and embryogenesis of Rhodnius prolixus. Parasitology. 2016;143(12):1569-79.

73. Legan SK, Rebrin I, Mockett RJ, Radyuk SN, Klichko VI, Sohal RS, Orr WC. Overexpression of glucose-6-phosphate dehydrogenase extends the life span of Drosophila melanogaster. J Biol Chem. 2008:283(47):32492-9.

74. Wang CT, Chen YC, Wang YY, Huang MH, Yen TL, Li H, Liang CJ, Sang TK, Ciou SC, Yuh $\mathrm{CH}$, et al. Reduced neuronal expression of ribose-5phosphate isomerase enhances tolerance to oxidative stress, extends lifespan, and attenuates polyglutamine toxicity in Drosophila. Aging Cell. 2012;11(1):93-103.

75. Bennett CF, Kwon JJ, Chen C, Russell J, Acosta K, Burnaevskiy N, Crane MM, Bitto A, Vander Wende $\mathrm{H}$, Simko M, et al. Transaldolase inhibition impairs mitochondrial respiration and induces a starvation-like longevity response in Caenorhabditis elegans. PLoS Genet. 2017;13(3):e1006695.

76. Dayton TL, Jacks T, Vander Heiden MG. PKM2, cancer metabolism, and the road ahead. EMBO Rep. 2016;17(12):1721-30.

77. Milenkovic D, Blaza JN, Larsson NG, Hirst J. The enigma of the respiratory chain Supercomplex. Cell Metab. 2017:25(4):765-76.

78. Scialo F, Sriram A, Fernandez-Ayala D, Gubina N, Lohmus M, Nelson G, Logan A, Cooper HM, Navas P, Enriquez JA, et al. Mitochondrial ROS produced via reverse Electron transport extend animal lifespan. Cell Metab. 2016:23(4):725-34

79. Copeland JM, Cho J, Lo T Jr, Hur JH, Bahadorani S, Arabyan T, Rabie J, Soh J, Walker DW. Extension of Drosophila life span by RNAi of the mitochondrial respiratory chain. Current Biol. 2009;19(19):1591-8. 
80. Yang $Y, X u$ S, Xu J, Guo Y, Yang G. Adaptive evolution of mitochondrial energy metabolism genes associated with increased energy demand in flying insects. PLoS One. 2014;9(6):e99120.

81. Leis M, Pereira MH, Casas J, Menu F, Lazzari CR. Haematophagy is costly: respiratory patterns and metabolism during feeding in Rhodnius prolixus. J Exp Biol. 2016;219(Pt 12):1820-6.

82. Nogueira NP, Saraiva FM, Sultano PE, Cunha PR, Laranja GA, Justo GA, Sabino KC, Coelho MG, Rossini A, Atella GC, et al. Proliferation and differentiation of Trypanosoma cruzi inside its vector have a new trigger: redox status. PLoS One. 2015;10(2):e0116712.

83. Zumaya-Estrada FA, Martinez-Barnetche J, Lavore A, Rivera-Pomar R, Rodriguez MH. Comparative genomics analysis of triatomines reveals common first line and inducible immunity-related genes and the absence of Imd canonical components among hemimetabolous arthropods. Parasit Vectors. 2018;11(1):48.

84. Schmieder R, Edwards R. Quality control and preprocessing of metagenomic datasets. Bioinformatics. 2011;27(6):863-4.

85. Giraldo-Calderon GI, Emrich SJ, MacCallum RM, Maslen G, Dialynas E, Topalis P, Ho N, Gesing S, VectorBase C, Madey G, et al. VectorBase: an updated bioinformatics resource for invertebrate vectors and other organisms related with human diseases. Nucleic Acids Res. 2015;43(Database issue):D707-13.

86. Adams MD, Celniker SE, Holt RA, Evans CA, Gocayne JD, Amanatides PG, Scherer SE, Li PW, Hoskins RA, Galle RF, et al. The genome sequence of Drosophila melanogaster. Science. 2000;287(5461):2185-95.

87. Gene Ontology C. Gene ontology consortium: going forward. Nucleic Acids Res. 2015:43(Database issue):D1049-56.

88. Rivals I, Personnaz L, Taing L, Potier MC. Enrichment or depletion of a GO category within a class of genes: which test? Bioinformatics. 2007;23(4):401-7.

89. Finn RD, Bateman A, Clements J, Coggill P, Eberhardt RY, Eddy SR, Heger A, Hetherington K, Holm L, Mistry J, et al. Pfam: the protein families database. Nucleic Acids Res. 2014;42(Database issue):D222-30.

90. Eisen MB, Spellman PT, Brown PO, Botstein D. Cluster analysis and display of genome-wide expression patterns. Proc Natl Acad Sci U S A. 1998;95(25):14863-8.

91. Nei M, Gojobori T. Simple methods for estimating the numbers of synonymous and nonsynonymous nucleotide substitutions. Mol Biol Evol. 1986;3(5):418-26.

92. Muse SV, Gaut BS. A likelihood approach for comparing synonymous and nonsynonymous nucleotide substitution rates, with application to the chloroplast genome. Mol Biol Evol. 1994;11(5):715-24.

93. Felsenstein J. Evolutionary trees from DNA sequences: a maximum likelihood approach. J Mol Evol. 1981;17(6):368-76.

94. Kumar S, Stecher G, Tamura K. MEGA7: molecular evolutionary genetics analysis version 7.0 for bigger datasets. Mol Biol Evol. 2016;33(7):1870-4.

\section{Ready to submit your research? Choose BMC and benefit from:}

- fast, convenient online submission

- thorough peer review by experienced researchers in your field

- rapid publication on acceptance

- support for research data, including large and complex data types

- gold Open Access which fosters wider collaboration and increased citations

- maximum visibility for your research: over $100 \mathrm{M}$ website views per year

At BMC, research is always in progress.

Learn more biomedcentral.com/submissions 